\title{
Passive Radar DPCA Schemes with Adaptive Channel Calibration
}

\author{
Giovanni Paolo Blasone, Student Member, IEEE, Fabiola Colone, Senior Member, IEEE, Pierfrancesco \\ Lombardo, Senior Member, IEEE, Philipp Wojaczek, Member, IEEE, Diego Cristallini, Member, IEEE
}

\begin{abstract}
This paper addresses the problem of direct signal interference (DSI) and clutter cancellation for passive radar systems on moving platforms employing displaced phase centre antenna (DPCA) approach. Attention is focused on the development of signal processing strategies able to compensate for the limitations deriving from amplitude and phase imbalances that affect the two channels employed on receive. First, we show that using the signal received from the illuminator of opportunity as a source for channels calibration might be ineffective when DSI and clutter echoes have different directions of arrival, due to the effect of angle-dependent channel imbalance. Then, a two-stage strategy is proposed, consisting of a preliminary DSI removal stage at each receive channel, followed by a clutter-based calibration approach that basically enables an effective DPCA clutter suppression. Different strategies for channel calibration are proposed, aimed at compensating for potential angle and range dependent channel errors, based on the maximization of the cancellation performance. Effectiveness of this scheme is shown against experimental data from a DVB-T based moving passive radar, in the presence of both real and synthetic moving targets.
\end{abstract}

Index Terms-passive radar, PCL, DPCA, STAP, GMTI, clutter cancellation, direct signal interference, channel calibration.

\section{INTRODUCTION}

In the last years, passive radar (or passive coherent location - PCL) systems have been gaining considerable and increasing attention in the scientific community and recent developments have opened new challenging areas of research and applications, [1]-[3]. Currently, several research groups are investigating the capabilities of PCL receivers mounted on moving platforms, for the purpose of target detection and ground imaging, [4]-[10].

Passive radar has reached a state of relative maturity for fixed platform operation. Stationary sensors have widely proved their ability to detect and localize air and ground targets exploiting the signals transmitted by illuminators of opportunity. In the future, this ability will also be available for mobile platforms. Mobile PCL receivers may offer a number of strategic advantages and extended functionalities compared to stationary ground-based solutions, which make them attractive for different kind of applications, such as ground moving target indication (GMTI) and synthetic aperture radar (SAR). These benefits are paid by the presence of signal distortions caused by the Doppler effect, which can adversely affect system performance: (i) the frequencyshifted reference signal must be reconstructed independently of motion, and (ii) the Doppler spectrum of the clutter echoes

G. P. Blasone, F. Colone and P. Lombardo are with the Dept. of Information Engineering, Electronics and Telecommunications (DIET), Sapienza University of Rome, Italy, (e-mail: \{giovannipaolo.blasone, fabiola.colone, pierfrancesco.lombardo\}@uniroma1.it) is spread by the relative motion of receiver with respect to the stationary scene. The latter effect, well known for standard airborne radar, tends to be even more stressed at the VHF/UHF bands of the widely used FM radio and DVB-T illuminators of opportunity, because of the wide antenna beams typically available. The detection of slow-moving targets requires a proper suppression of the clutter echoes, which is usually obtained by applying Space-Time Adaptive Processing (STAP) to the signals collected by multiple onboard receiving channels, connected to antennas with some along track displacement. The desirable low-cost characteristic of passive radar, the typical size of antennas at VHF/UHF bands and the high data rate of digital broadcast transmissions suggest the use of only few spatial channels and a simple processing scheme. For this reason, a displaced phase centre antenna (DPCA) approach has been primarily considered in [5]-[10].

Conventional DPCA performs a non-adaptive subtraction of radar echoes collected by two along-track displaced receiving antennas at the times that their two-way phase centres occupy the same spatial position [14]. Echoes from stationary background are ideally cancelled out, being the performance only limited by thermal noise, internal clutter motion, waveform variability, and possible channel imbalance. Conversely, echoes from moving targets, shifted in phase due to their own radial motion, are preserved and can be ideally detected. Notice that DPCA and more in general space-time processing intended for passive radar require the availability of at least two surveillance channels; reference signal can be whether reconstructed from one of them or received by a dedicated reference channel.

A first proof of concept of mobile passive radar is given in [4], where an airborne PCL using FM transmission was presented. The authors in [5] and [6] consider the use of DPCA to suppress clutter returns against experimental data from an airborne FM-based passive radar and against simulated DVB-T data. The effects of signal mismatches on the correlation process for a moving passive radar are analysed in [7], while [8] investigates the effects of motion induced Doppler shift on reference signal reconstruction.

In [10] a processing scheme is proposed, based on a reciprocal range compression filter in conjunction with a flexible DPCA approach, which removes the performance limitations deriving from the uncontrolled waveform variability. Its effectiveness is proved for a DVB-T based PCL system against both simulated and experimental data.

P. Wojaczek and D. Cristallini are with Fraunhofer Institute for HighFrequency Physics and Radar Techniques FHR, Wachtberg, Germany, (email: \{philipp.wojaczek, diego.cristallini\} @ fhr.fraunhofer.de)

(Corresponding author: G. P. Blasone) 
As known, DPCA poses strong constraints on antenna alignment and is intrinsically sensitive to imbalance between receiving channels, which have to be identical to provide an effective suppression of stationary scene returns. For this reason, the scheme in [10] includes a simple approach for channel calibration, which compensates for amplitude and phase inequalities by exploiting the direct signal contribution from the transmitter, assumed as a strong and reliable source. In principle, DSI can be seen as a stationary return and DPCA should suppress it together with the other clutter components.

Following the preliminary analysis in [12] and [13], this paper shows that channels imbalance can be angle dependent due to non-identical receiving antenna patterns (especially outside the main lobe region). This might be a critical problem in PCL systems, typically employing low directivity antennas, especially when clutter echoes and DSI come from different directions of arrival (DoA). In this case, the simple calibration proposed in [10] can be ineffective and alternative solutions should be found.

Channel imbalance is a well-known issue in conventional active radar. Several studies are present in literature analysing the role of channel mismatch in the context of array beamforming and space-time processing [14]-[15]. For specific kind of applications, such as clutter cancellation for GMTI, accurate factory or in-field calibration might be not feasible or not sufficient. Methods for array self-calibration based on received signals have been studied [16]-[20], as well as STAP techniques in presence of steering vector mismatch [21]-[23]. Specific approaches have been developed for the case of SAR-GMTI [33]-[36], which take advantage of the angle-Doppler dependence.

We aim at developing ad-hoc solutions for the passive radar case, when using a DPCA approach for clutter cancellation. In this situation, we need to tackle all the critical aspects brought in by the passive bistatic operation, while preserving the low-cost approach provided by the use of the DPCA technique. Among the critical aspects, the use of commercial-off-the-shelf components and the wavelength of typically exploited signals of opportunity may pose some limitations to preliminary system calibration. Moreover, low directivity antennas, typical sidelobe level, and bistatic geometry characterized by broadcast transmissions determine the need for an accurate channel calibration on a very wide angular sector in order to achieve good clutter cancellation, especially for essentially non-adaptive approaches like DPCA. This is exacerbated by the presence of strong continuous-wave direct signal components coming from the illuminator of opportunity and by its multipath replicas. However, the typical passive radar operates with very long integration times, which potentially provide a high Doppler frequency resolution and in turn a good angular discrimination capability.

Different strategies for digital channel calibration are proposed, operating directly on the range-Doppler maps and based on clutter contributions. Starting from the estimation of a single calibration coefficient, the flexibility of the calibration model is progressively increased, in order to compensate for potential angle and range dependent channel errors, thus maximizing the resulting cancellation capability at the output of DPCA subtraction.
The effectiveness of the considered processing scheme, along with the channel calibration approaches, is investigated against experimental data from a DVB-T based PCL system mounted on a ground-based moving vehicle. The performance is analysed in terms of disturbance cancellation capability, as well as in terms of signal-to-clutter-plus-noise ratio (SCNR) improvement against synthetic targets. In addition, the results are reported for a real cooperative aerial target employed during the experimental campaign.

The paper is organized as follows. In Section II, signal model and processing scheme from [10] are briefly recalled. Section III illustrates the acquisition campaign and the experimental dataset used and gives evidence of the limits of a channel calibration based on DSI. In Section IV, we introduce a two-stage approach for combined suppression of DSI and clutter, resorting to a time domain cancellation technique prior to the DPCA stage. In Section V, adaptive clutter-based strategies for channel calibration are presented, with increasing degrees of freedom, aimed at maximizing cancellation performance, by compensating for angle and range dependent channel errors. Since the increase of range/angle localization makes the adaptation more sensitive to the presence of interfering targets, a robust scheme is also introduced. Results obtained in terms of SCNR improvement against both real and simulated moving targets are reported in Section VI. Finally, conclusions are drawn in Section VII.

\section{SIGNAL MODEL AND PROCESSING SCHEME}

\section{A. System geometry and signal model}

We consider a passive radar receiver mounted on a moving platform that exploits a ground-based transmitter as illuminator of opportunity (see Fig. 1). The platform moves at constant velocity $v_{p}$ on a straight line. Two parallel receiving channels are available, displaced by $d$ in the alongtrack direction, in a side-looking configuration. They are referred to as leading antenna (LA) and trailing antenna (TA).

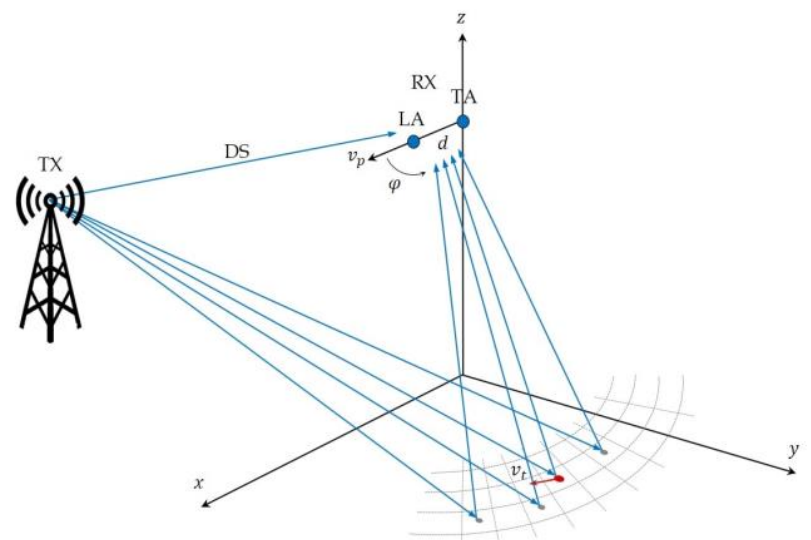

Fig. 1. System geometry for a mobile passive radar exploiting a groundbased transmitter as illuminator of opportunity.

By explicitly including the antenna pattern in the signal model adopted in [10], the discrete time baseband signal representing the clutter contribution at the two antennas can be expressed as the superposition of echoes from stationary 
scatterers at different bistatic ranges $R_{q}\left(q=1, \ldots, N_{R}\right)$ and different angles $\varphi$ :

$$
\begin{gathered}
r_{C}^{(L A)}[l]=\sum_{q=1}^{N_{R}} \int_{\phi} G_{q}^{(L A)}(\varphi) A_{q}(\varphi) \sum_{n} s_{n}\left[l-n L-l_{\tau_{q}}\right] \\
\cdot e^{j 2 \pi \frac{v_{p}}{\lambda} \cos \varphi n T} d \varphi \\
r_{C}^{(T A)}[l]=\sum_{q=1}^{N_{R}} \int_{\phi} G_{q}^{(T A)}(\varphi) A_{q}(\varphi) \sum_{n} s_{n}\left[l-n L-l_{\tau_{q}}\right] \\
\cdot e^{j 2 \pi \frac{v_{p}}{\lambda} \cos \varphi n T} e^{-j 2 \pi \frac{d}{\lambda} \cos \varphi} d \varphi
\end{gathered}
$$

where

- $\varphi$ is the angle between the platform velocity vector and the receiver to scatterer line of sight;

- $A_{q}(\varphi)$ and $\tau_{q}=l_{\tau_{q}} / f_{s}$ are respectively the complex amplitude and bistatic propagation delay of echo from clutter patch at angle $\varphi$ and range $R_{q}, f_{s}$ being the sampling frequency;

- $G_{q}^{(L A)}(\varphi)$ and $G_{q}^{(T A)}(\varphi)$ are the complex amplitude gains of the LA and TA channels respectively; they represent the overall receiver chains, there including the antenna patterns, and encode possible amplitude and phase imbalance between the two channels;

- $f_{D}(\varphi)=\frac{v_{p}}{\lambda} \cos \varphi$ is the bistatic Doppler frequency of the generic clutter patch at angle $\varphi, \lambda$ being the signal carrier wavelength;

- transmitted signal has been partitioned in fragments of duration $T$ and $s_{n}[l]$ is the $n$-th fragment, including $L=$ $T f_{s}$ samples; the Doppler induced phase term within each fragment has been neglected.

Notice that the expressions above might include also the direct signal contribution from the exploited transmitter.

\section{B. DPCA processing scheme}

The considered processing scheme, originally presented in [10] for a DVB-T based passive radar, is sketched in Fig. 2. After a pre-processing stage, including synchronization to the transmitter and demodulation/remodulation of the reference DVB-T signal, the bistatic range-Doppler map is evaluated by means of a batches processing strategy:

i. the received signal is subdivided into short consecutive batches of duration $T$, deliberately selected to be equal to single OFDM symbols;

ii. range compression is performed on a batch-by-batch basis, using the reconstructed version of the reference signal and implementing a reciprocal filtering strategy;

iii. consecutive batches are coherently combined by means of a discrete Fourier transform (DFT) to synthesize the Doppler dimension.

The batches processing architecture significantly reduces the computational load in passive radar exploiting wideband digital waveform. Moreover, it recreates the conventional fast-time/slow-time framework of a pulsed radar operating at an equivalent PRF given by the inverse of the batch length $(P R F=1 / T)$.
The use of a reciprocal filter at the range compression stage has a twofold objective. On one hand, it allows to control the sidelobes level and mitigate undesired structure and grating lobes arising in the signal ambiguity function, which may hinder the detection of target echoes, especially when OFDM transmissions are exploited [24]-[26]. On the other hand, it yields significant advantages for the subsequent DPCA stage [10]. In fact, at the expense of a limited and predictable loss compared to the matched filter, the reciprocal filter allows to remove the temporal variability of the batch impulse response, due to the time-varying characteristics of the employed waveform of opportunity, so that an ideal clutter cancellation can be in principle obtained based on subsequent observations of a stationary scene. Notice that, in order to benefit from the two advantages above, a perfect reconstruction of the reference signal should be made available via the mentioned decode/recode approach. This intrinsically removes the effect of the multipath contributions to the reference signal.

A DPCA stage is then applied by resorting to a flexible technique, which allows to relax the constraint posed on the equivalent PRF. For bistatic radar employing a stationary transmitter, DPCA condition is given by:

$$
T_{D P C A}=K \cdot P R I=K \cdot T=d / v_{p}
$$

where $K$ is the integer number of symbols after which the two-way phase centres occupy the same position. Since this condition is hardly verified in real environment, in flexibleDPCA technique, the required time shift is performed in two steps: a coarse delay $T_{q}$ quantized to the equivalent PRI is applied in time domain; a residual fine delay $T_{f}$ is compensated in frequency domain by a linear phase term. In detail:

$$
T_{q}=\left\lfloor\frac{d}{v_{p} T}\right\rfloor T
$$

$$
T_{f}=T_{D P C A}-T_{q}
$$

The platform velocity must be measured by means of an inertial measurement unit (IMU) or estimated from the data. Based on this strategy, DPCA condition can be effectively established also when a batches architecture is adopted.

The described processing scheme should in principle provide an ideal cancellation of stationary background, by means of a simple subtraction of the delayed observations from the two channels, provided that the following conditions are met:

- perfect DPCA condition established by compensation of time delay according to (3);

- negligible internal clutter motion (ICM), i.e. constant amplitude $A_{q}(\varphi)$ within the processing interval;

- compensation of potential amplitude and/or phase imbalance between receiving channels.

Therefore, even assuming the first two conditions, DPCA performance strongly relies on the adoption of a proper calibration strategy before channel subtraction (see calibration block in Fig. 2). 


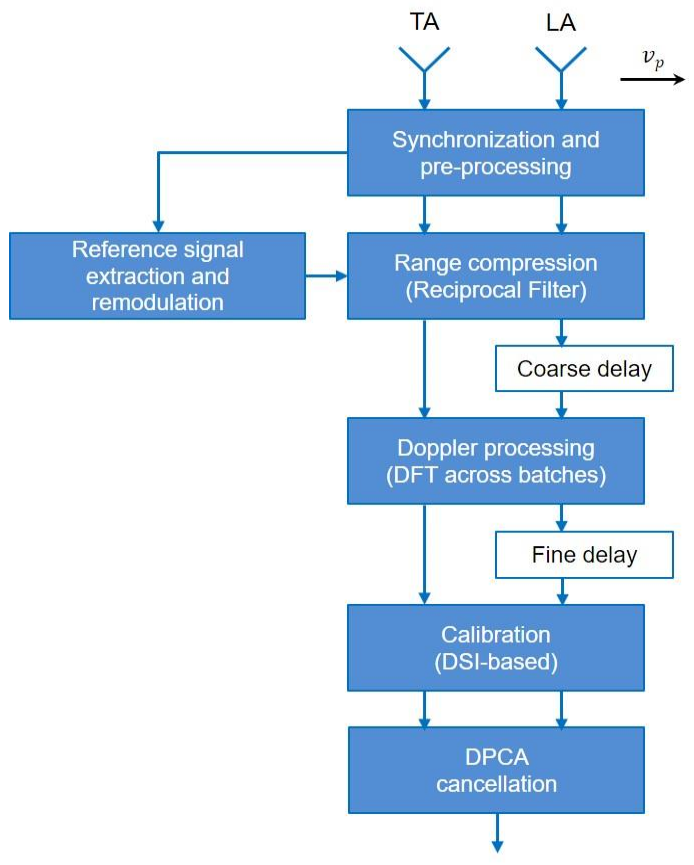

Fig. 2. Sketch of the flexible DPCA processing scheme as proposed in [10].

Starting from the signal model in (1) and following the same formalism adopted in [10], after range compression by reciprocal filtering, delay compensation and Doppler processing, the clutter contribution at the generic rangeDoppler bin $[l, m]$ of LA and TA channels can be expressed as follows:

$$
\begin{gathered}
z_{C}^{(L A)}[l, m]=\sum_{q=1}^{N_{R}} \int_{\phi} G_{q}^{(L A)}(\varphi) A_{q}(\varphi) g_{r}\left[l-l_{\tau_{q}}\right] \\
\cdot g_{d}\left[\frac{m}{N T}-\frac{v_{p}}{\lambda} \cos \varphi\right] d \varphi \\
z_{C}^{(T A)}[l, m]=\sum_{q=1}^{N_{R}} \int_{\phi} G_{q}^{(T A)}(\varphi) A_{q}(\varphi) g_{r}\left[l-l_{\tau_{q}}\right] \\
\cdot g_{d}\left[\frac{m}{N T}-\frac{v_{p}}{\lambda} \cos \varphi\right] d \varphi
\end{gathered}
$$

where $g_{r}[l]$ denotes the output of reciprocal range compression filter, equal for each signal batch, and $g_{d}[m]$ denotes the output of Doppler processing over $N$ consecutive batches (equal to a digital sinc function in case of uniform windowing [10]). Notice that generic cell $[l, m]$ is characterized by the superposition of clutter echoes from different range cells and different angular positions.

As apparent, clutter cancellation achievable by subtraction of the two range-Doppler maps in (4) would be hindered by the imbalance between receiving channels $\Gamma_{q}(\varphi)=G_{q}^{(T A)}(\varphi) / G_{q}^{(L A)}(\varphi)$.

The simplest model for channel calibration is based on the assumption of an angle-invariant amplitude and phase channel imbalance, namely $\Gamma_{q}(\varphi)=\Gamma_{0}$, that can be easily compensated for by applying a single complex multiplicative coefficient at one of the two channels output. This was basically the model adopted in [10], where the sought coefficient was evaluated based on the DSI contribution.
However, in the following, we show that such a simplifying hypothesis, in some cases, does not guarantee an effective calibration of the receiving channels, hence more realistic models will be adopted for the channels imbalance by properly taking into account the angle dependency.

\section{EXPERIMENTAL DATA AND LIMITATIONS OF DSI-BASED CHANNEL CALIBRATION}

\section{A. Overview of the acquisition campaign}

The processing schemes and channel calibration strategies proposed in this paper are tested against a set of experimental data acquired by a DVB-T based multichannel PCL system mounted on a ground moving platform.

The acquisition campaign was conducted by Fraunhofer FHR in a rural area of the Eifel region, in western Germany. The selected DVB-T illuminator of opportunity was the Eifel/Scharteberg transmitter. The PCL system consisted of four receiving channels, serving as surveillance channels, connected to discone antennas (omnidirectional in azimuth) and displaced in the along-track direction. As receivers two Parasol units, designed by Fraunhofer FHR, were employed, each providing two receiving channels [27]. Radiation absorbing material (RAM) was placed on one side, in order to attenuate back lobes contributions and thus forming a single side-looking configuration. The system was mounted on a trailer behind a van (see Fig. 3). Table I summarizes the parameters of exploited DVB-T transmission and the main acquisition and processing parameters.

For the purpose of our study, signals collected by two out of the four receiving channels are exploited, in order to analyse the effects of different calibration strategies with the considered DPCA scheme. Specifically, channels associated to adjacent antenna pairs are considered. The reference signal is reconstructed from one of the surveillance channels.

The considered case study is characterized by a bistatic geometry where the transmitter is located approximately in the direction opposite to the observed scene, with direct signal impinging on the antenna back-lobes (see Fig. 4(a) for a sketch of the acquisition geometry).

\begin{tabular}{|c|c|c|}
\hline Symbol & Description & Value \\
\hline \multicolumn{3}{|c|}{ DVB-T signal parameters } \\
\hline & DVB-T Standard & 8k 16QAM \\
\hline$f_{c}$ & Carrier frequency & $690 \mathrm{MHz}$ \\
\hline$N_{c}$ & Number of useful carriers & 6817 \\
\hline$T_{u}$ & Useful symbol duration & 896 us \\
\hline$T_{g}$ & Guard interval duration & 224 us \\
\hline$T_{S}$ & OFDM symbol duration & 1120 us \\
\hline$B$ & Bandwidth & $7.61 \mathrm{MHz}$ \\
\hline \multicolumn{3}{|c|}{ System and processing parameters } \\
\hline$v_{p}$ & Platform velocity & $\approx 13.8 \mathrm{~m} / \mathrm{s}$ \\
\hline$d_{a}$ & Antenna spacing & $0.36 \mathrm{~m}$ \\
\hline CPI & Coherent processing interval & $512 \mathrm{~T}_{\mathrm{s}}$ \\
\hline
\end{tabular}

An ultralight aircraft from Fraunhofer FHR (Delphin) has been employed as a cooperative target during the acquisition.

TABlE I. PARAMETERS OF EXPERIMENTAL TEST 


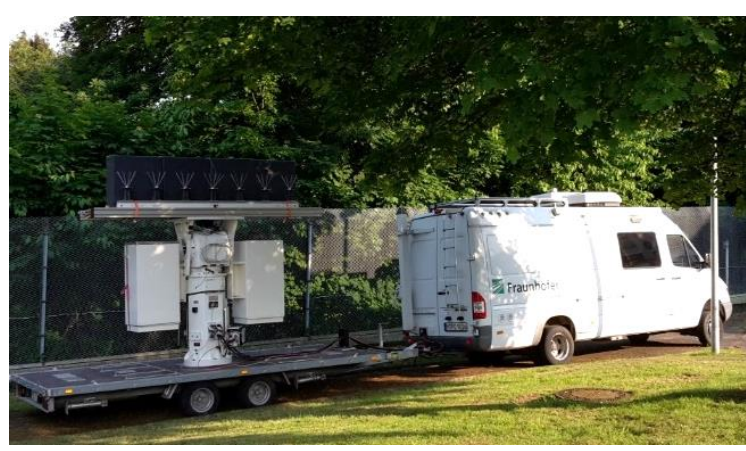

(a)

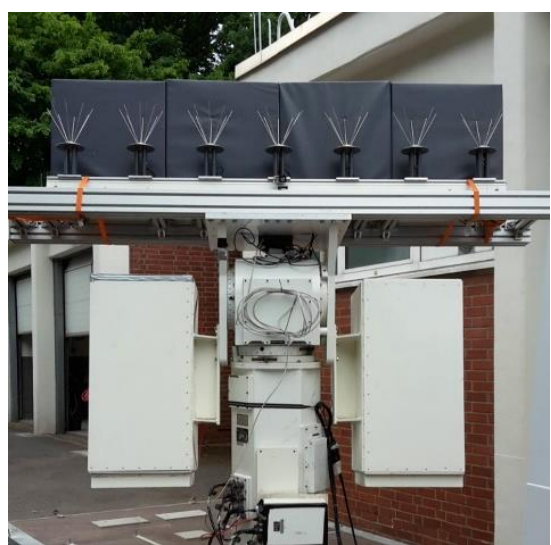

(b)

Fig. 3. The multichannel PCL receiver mounted on the back of a van in a side-looking configuration (a). Detailed view of receive array (b).

\section{B. Analysis of data}

An example of range-Doppler map obtained from a single channel, namely before DPCA, is reported in Fig. 4(b). Specifically, a coherent processing interval (CPI) of $512 T_{s} \cong$ $0.57 s$ is considered and a Taylor windowing is applied in both range and Doppler domain, in order to limit the sidelobes level of the ambiguity function down to $-40 \mathrm{~dB}$ below its main peak. Notice that the resulting range-Doppler map is oversampled and scaled to the estimated noise power level.

A strong DSI contribution appears at the first bistatic range bin and at Doppler frequency $-3.3 \mathrm{~Hz}$; this low Doppler value is due to the angle formed by the Rx-Tx line of sight (LOS) and the platform velocity vector, which is close to 90 deg. in the considered data file (see Fig. 4(a)).

Given the platform velocity and the employed antennas, echoes from the stationary scene extend in Doppler over a bandwidth of approximately $\pm v_{p} / \lambda \cong \pm 32 \mathrm{~Hz}$ and are characterized by a strong heterogeneity in terms of power levels across the map (wide dynamic range); also, we observe the presence of large clutter discretes. In the reported map, the bistatic range is limited to the first $8 \mathrm{~km}$, since in this acquisition few appreciable clutter contributions are present beyond that limit due to the terrain conformation (the PCL system was operated in a hilly region).

By comparing the obtained range-Doppler map with the optical image in Fig. 4(a), a few distributions of strong scatterers can be easily identified. Notice that, in Fig. 4(b), these are indicated by coloured lines obtained by projecting onto the bistatic domain the curved lines in homologous colours in Fig. 4(a). This analysis allows to better understand the characteristics of the clutter scene under consideration, as well as intrinsic limitations to the cancellation capability.

It is worth noting that the strong clutter contributions mainly arise from densely vegetated areas. In fact, the lines in Fig. 4(a) typically lie on the perimeter of such areas, being the first line of trees responsible of the strongest returns.

Assuming, for the sake of simplicity, a Gaussian model for windblown clutter power spectral density, typical rms spectral width from 0.1 to $0.3 \mathrm{~m} / \mathrm{s}$ are obtained [28], corresponding to wind speed from 1 to $30 \mathrm{mph}$. This would result in a clutter correlation coefficient in the order of $\rho\left(T_{D P C A}\right)=0.975-0.997$ for the case under consideration. Therefore, to a first approximation, clutter attenuation performance of a single canceller is expected to be limited to $C A=\left[0.5 /\left(1-\operatorname{Re}\left\{\rho\left(T_{D P C A}\right)\right\}\right] \cong 13-22 d B\right.$ due to the sole effect of ICM [29].

Importantly enough, especially in the first kilometre, returns are affected by a superposition of front and back clutter echoes. Moreover, the area is characterized by the presence of wind turbines, located in the back-lobe direction and indicated with a red cross in Fig. 4(b). The cooperative aerial target, whose expected position is indicated by a white ellipse in Fig. 4(b), appears as buried into clutter background.

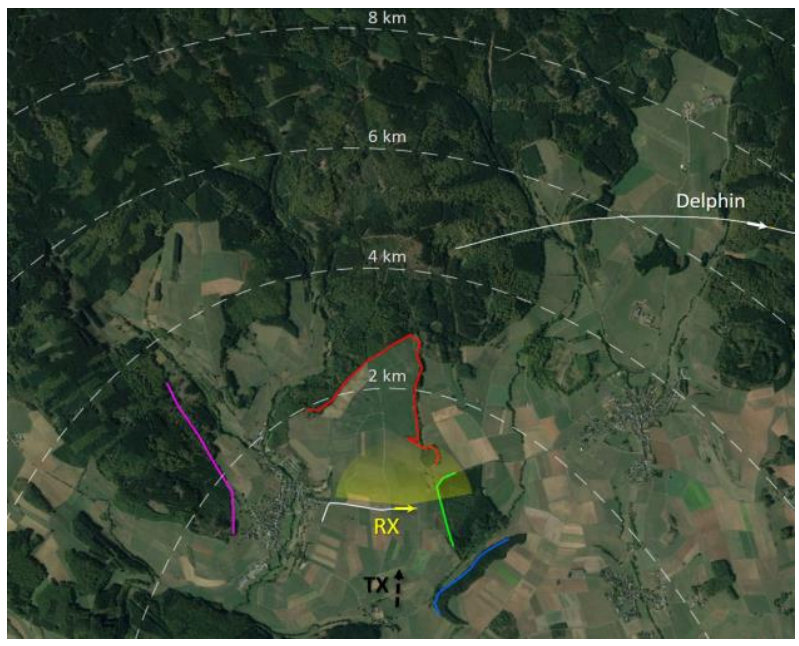

(a)

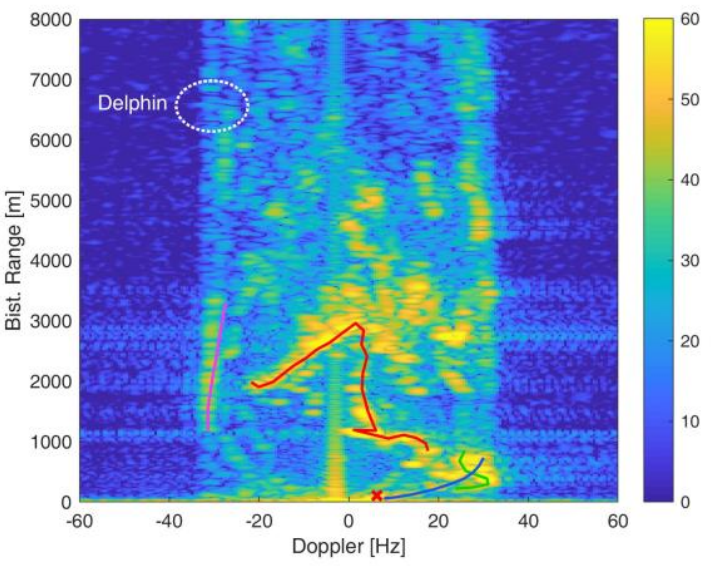

(b)

Fig. 4. Analysis of clutter contributions from the area surrounding the PCL system during the considered acquisition: (a) optical image with indication of the TX DoA and the RX and target position and direction of motion; (b) corresponding single channel range-Doppler map. Coloured lines indicate some distributions of strong scatterers mapped into the bistatic domain. 
() 2020 IEEE. Personal use of this material is permitted. Permission from IEEE must be obtained for all other uses, in any current or future media, including reprinting/republishing this material for advertising or promotional purposes, creating new collective works, for resale or redistribution to servers or lists, or reuse of any copyrighted component of this work in other works. DOI: 10.1109/TAES.2020.2987478.

\section{Results after channels calibration based on direct signal}

In [10], the simplest approach was adopted for channel calibration, based on the direct signal coming from the exploited transmitter. A single complex coefficient is evaluated at the range-Doppler bin corresponding to the DSI, to compensate for channel imbalance, which is assumed to be angle invariant, i.e. $\Gamma(\varphi)=\Gamma_{0}$. Specifically, the complex correction coefficient is evaluated as:

$$
\widehat{\Gamma_{0}}=\frac{z^{(T A)}\left[l_{t x}, m_{t x}\right]}{z^{(L A)}\left[l_{t x}, m_{t x}\right]}
$$

where $z^{(\gamma)}\left[l_{t x}, m_{t x}\right]$ is the complex value of range-Doppler map at location corresponding to the direct signal for the $\gamma=$ 'TA' /'LA' channel. This approach can rely on a strong and reliable source and proved to be effective in the analysis conducted in [10]-[11], where a dominant DSI contribution was present impinging on the main-lobe of the receiver antennas.

Unfortunately, under more general conditions, the direct signal is not representative of the overall clutter distribution in terms of amplitude and phase calibration requirements. This might be the case when the DSI DoA is not within the antenna main beam, but rather it impinges on the sidelobes or the back-lobes regions, where the patterns of the employed antennas are likely to differ.

This is shown in Fig. 5 that reports the results of the application of the flexible-DPCA scheme to the selected data file, when adopting the DSI-based calibration strategy against a DSI signal impinging on the receiver antenna backlobes. Notice that all range-Doppler maps are scaled so that $0 \mathrm{~dB}$ corresponds to the estimated noise power level, to allow a direct comparison of results. By comparing Fig. 4(b) and Fig. 5, it is evident how cancellation effectively occurs only for the DSI contribution, while echoes from stationary scene are only slightly attenuated or partially suppressed.

In order to measure the effectiveness of clutter suppression and compare the results of different approaches, we resort to the cancellation ratio (CR), which expresses the attenuation in clutter power provided by the DPCA stage. It is defined as:

$$
C R[l, m]=\frac{P_{c}^{\text {in }}[l, m]}{P_{c}^{\text {out }}[l, m]}
$$

where $P_{c}^{i n}[l, m]$ is the clutter power measured at the generic delay-Doppler bin of maps obtained at the single channels (an average value is considered between the LA and TA channels) and $P_{c}^{\text {out }}[l, m]$ is that measured after the application of DPCA.

Fig. 6 shows the CR map obtained for the result in Fig. 5 . As expected, perfect cancellation is obtained for the rangeDoppler bin corresponding to DSI and high CR values are observed for the corresponding sidelobes contributions. Notice that the reported CR map has been upper limited to 20 $\mathrm{dB}$, to facilitate the analysis of the small CR values; however, the CR values obtained at the DSI sidelobes are typically higher. Conversely, the average power reduction measured in the area corresponding to the overall endo-clutter region is

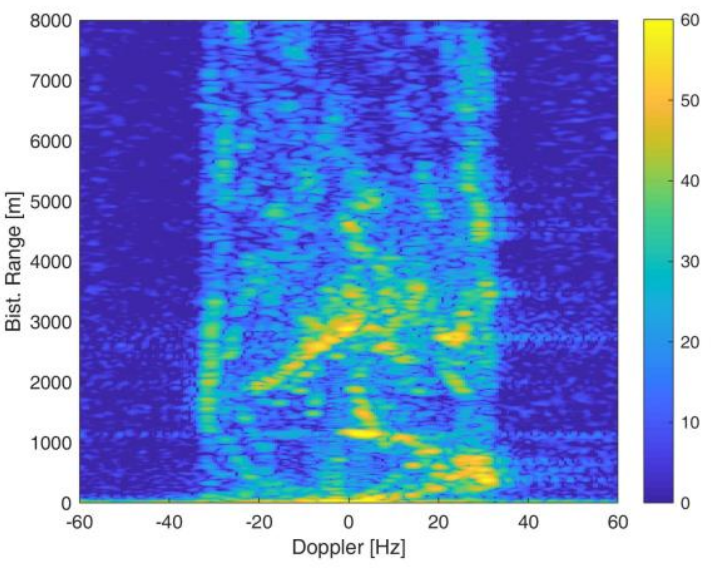

Fig. 5. Range-Doppler map after DPCA with DSI-based calibration.

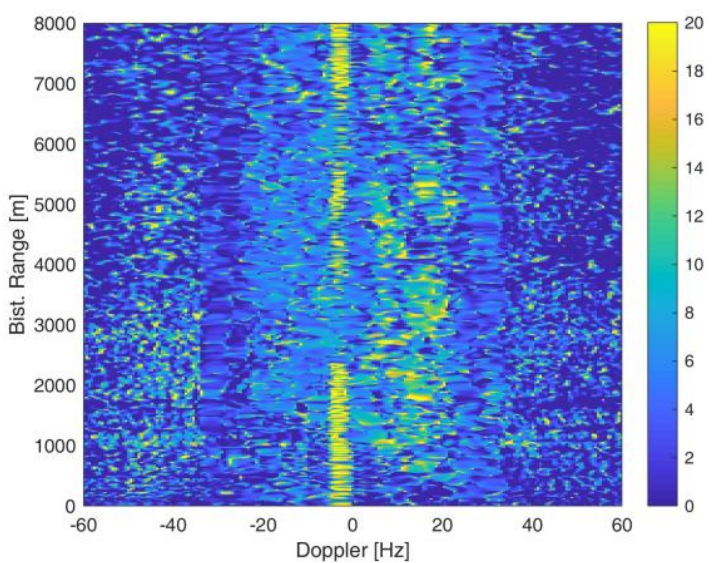

Fig. 6. Cancellation Ratio obtained after DPCA with DSI-based calibration.

less than $6 \mathrm{~dB}$, well below the predicted values according to the observed clutter characteristics, i.e. the expected ICM.

Such limitation could be in principle caused by a number of factors other than the ICM. However, in the following we show that mostly residual channel imbalance prevents the clutter echoes from being effectively cancelled. This result can be mainly attributed to angle-dependent differences in the patterns of the employed surveillance antennas and gives evidence of the limits of a calibration approach based on the DSI and, more in general, of the simplified model of a uniform inter-channel imbalance.

\section{TWO-STAGE CANCELLATION APPROACH}

The analysis reported in the previous section suggests the need for alternative calibration approaches based on clutter echoes instead of on DSI. However, the direct-path component and related side-lobes are largely overlapped with clutter contributions in the range-Doppler map. This may represent a major concern for imbalance estimation if the DSI is not effectively counteracted.

Therefore, in this section, we propose a two-stage cancellation approach, which allows for a combined suppression of DSI and clutter contributions. This approach was preliminarily considered in [13], where it was adopted in conjunction with a simple channel calibration strategy, as 
briefly summarized in the following. This two-stage approach is further extended in this paper to include more effective calibration strategies.

The idea is to firstly suppress the DSI on both surveillance channels in time domain, by resorting to extensive cancellation algorithm (ECA) techniques; then, clutter cancellation is performed in space-time domain via DPCA, after a clutter-based channel calibration. Accordingly, the resulting processing scheme is sketched in Fig. 7.

ECA techniques [30]-[32] are able to remove DSI by projecting the surveillance signal into a subspace orthogonal to the direct signal. We adopt in this case the ECA-CD (ECA by Carrier and Doppler) version of the algorithm [31], which operates carrier by carrier by exploiting the OFDM modulation of the considered waveforms. Specifically, we apply a proper Doppler shift to the available reference signal, in order to centre the disturbance removal filter on the estimated DSI Doppler bin. Additionally, the filter spans over a few adjacent Doppler bins in order to improve the suppression of DSI and associated sidelobes.

It is assumed that a significant difference in amplitude and phase channel imbalance $\Gamma(\varphi)$ exists between the DSI, typically dominant and coming from a specific direction, depending on the geometry, that might even correspond to the antenna back-lobes, and the main clutter background, mostly coming from a range of directions inside the antenna front lobe, where the imbalance is supposed to be almost invariant. Therefore, after DSI removal, channel calibration is performed based on clutter contributions appearing in the resulting range-Doppler maps, no longer affected by the direct signal component.

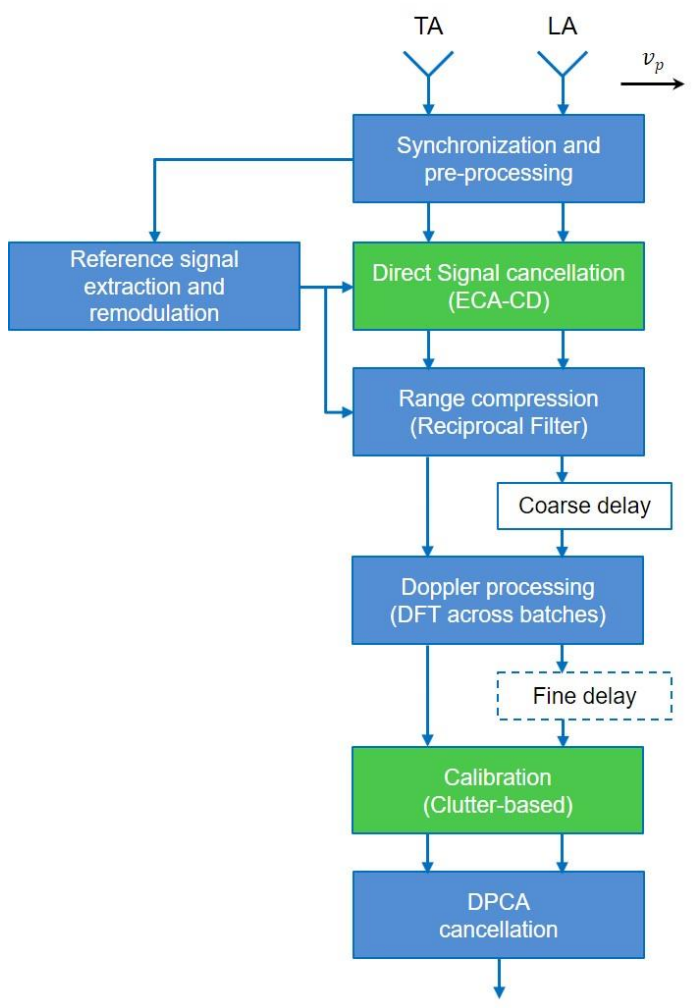

Fig. 7. Modified processing scheme implementing the proposed two-stage cancellation approach.
In [13], aiming at preserving the paradigm of a simplified approach, we considered the estimation of a single complex calibration coefficient maximizing clutter cancellation at the subsequent DPCA stage.

Starting from the model in (4), we assume that, after suppression of DSI, the remaining clutter contributions are affected by an approximately uniform imbalance, i.e. $G_{q}^{(T A)}(\varphi) / G_{q}^{(L A)}(\varphi)=\Gamma_{c}$. Therefore, for clutter signal at the output of the two range-Doppler maps, the following relation holds: $z_{c}^{(T A)}[l, m]=\Gamma_{c} \cdot z_{c}^{(L A)}[l, m]$.

Channel imbalance can then be estimated by resorting to a least square approach operated on the overall endo-clutter area. Specifically, we look for the calibration coefficient minimizing the power at the output of the DPCA subtraction:

$$
\begin{aligned}
\widehat{\Gamma_{c}} & =\underset{\Gamma}{\operatorname{argmin}}\left\{E\left\{\left|z^{(T A)}[l, m]-\Gamma \cdot z^{(L A)}[l, m]\right|^{2}\right\}\right\} \\
& =\frac{E\left\{z^{(T A)}[l, m] z^{(L A)^{*}}[l, m]\right\}}{E\left\{\left|z^{(L A)}[l, m]\right|^{2}\right\}}
\end{aligned}
$$

where $*$ indicates the complex conjugate and $z^{(\gamma)}[l, m]$ are the complex values of range-Doppler maps at the $\gamma=$ 'TA' /'LA' channel, including clutter and thermal noise. In this case, the TA channel is arbitrarily taken as reference and the LA channel is adjusted by multiplication with $\widehat{\Gamma_{c}}$.

In practice, the expected values in (7) are replaced by their estimates obtained over proper clutter regions. For instance, by selecting a rectangular clutter area with range extent limited between indexes $l_{1}$ and $l_{2}$ and Doppler extent limited between indexes $\mathrm{m}_{1}$ and $\mathrm{m}_{2}$, the calibration coefficient can be evaluated as:

$$
\widehat{\Gamma_{c}}=\frac{\sum_{l=l_{1}}^{l_{2}} \sum_{m=m_{1}}^{m_{2}} z^{(T A)}[l, m] z^{(L A)^{*}}[l, m]}{\sum_{l=l_{1}}^{l_{2}} \sum_{m=m_{1}}^{m_{2}}\left|z^{(L A)}[l, m]\right|^{2}}
$$

The presented two-stage approach allows to achieve a combined suppression of both DSI and clutter, despite adopting a strategy based on a single calibration coefficient (SCC), provided that this is estimated based on clutter contributions. The advantages of this approach are not only due to the addition of a dedicated cancellation stage devoted to the removal of the DSI but also to the mitigation of the effects of the DSI on the subsequent calibration stage. In fact, in [13] we have shown that a poorer cancellation capability is obtained against the stationary scene if the calibration coefficient is estimated before the removal of the DSI overlapping on the range-Doppler map.

Fig. 8 shows the range-Doppler map obtained after applying the two-stage cancellation scheme against the data set under consideration in this paper. Calibration is performed, according to (8), based on the overall endo-clutter region: bistatic range interval within $[150 ; 8000] \mathrm{m}$ and Doppler frequency $[-32 ; 32] \mathrm{Hz}$. The first few range cells are skipped in order for the calibration not to be affected by additional back lobes clutter echoes and the aforementioned wind turbines. The corresponding CR maps are reported in Fig. 9 and Fig. 10, for the ECA-CD stage and the final DPCA 
stage respectively, in order to address the effects of each cancellation stage separately.

As expected, the DSI is largely cancelled by the ECA-CD stage and clutter power is significantly attenuated by the following DPCA stage. Focusing on cancellation performance of the DPCA stage, a remarkable clutter attenuation is now achieved, with an average power reduction in the endo-clutter region that exceeds $14 \mathrm{~dB}$ and $\mathrm{CR}$ values up to $25 \mathrm{~dB}$ at specific range-Doppler locations.

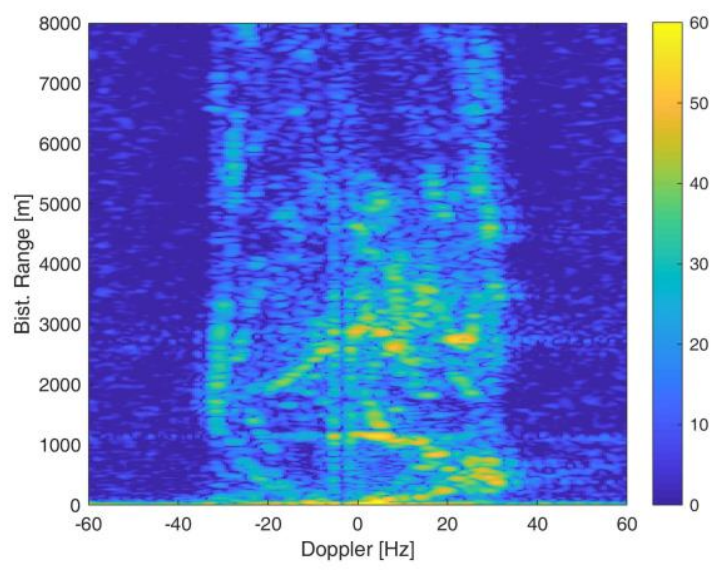

Fig. 8. Range-Doppler map after two-stage cancellation scheme with SCC approach.

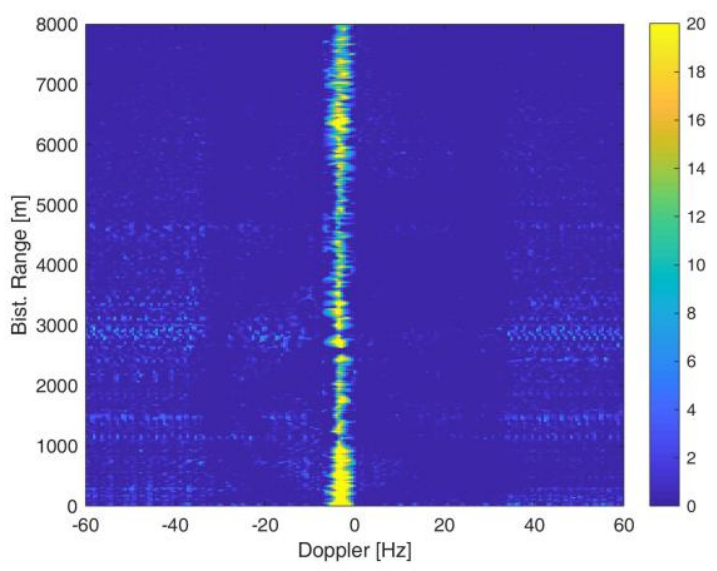

Fig. 9. Cancellation Ratio obtained after ECA-CD stage only.

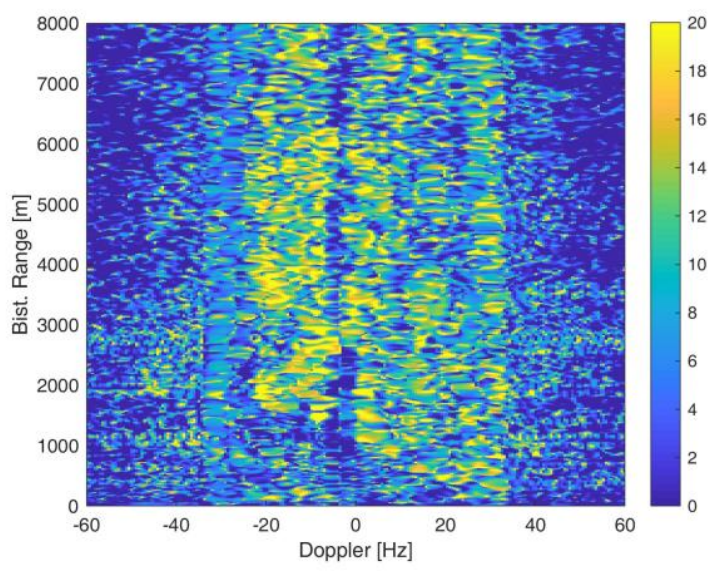

Fig. 10. Cancellation Ratio obtained after DPCA stage with SCC approach.
The residual uncancelled clutter power in the final map highlights the presence of further limitations to DPCA performance, potentially related to several effects, such as irregular platform motion, possible antenna misalignment and crabbing, mutual coupling effects, as well as the ICM.

However, by observing Fig. 10, one might notice that the CR values are much higher at specific Doppler intervals while they significantly reduce moving across the Doppler dimension. Unfortunately, this is the case of the Doppler region where the cooperative target Delphin lies and this does not allow to easily discriminate its echoes from the surrounding residual clutter.

As well known, for stationary scene echoes received by a moving radar, there is a direct mapping between Doppler frequency and azimuth angle of arrival. Based on this consideration, the above analysis suggests that additional angle-dependent channel imbalances should still be compensated for by resorting to more sophisticated calibration strategies, which will be introduced in the next section.

\section{ADAPTIVE CLUTTER-BASED CALIBRATION TECHNIQUES}

Several factors can influence the amplitude and phase response of receiving channels. Dissimilarities in antenna patterns, interaction with near-field obstacles, mutual coupling effect between array elements, and other potential sources of errors may cause non-negligible variations of channel imbalance as function of the angle of arrival. These variations can considerably limit DPCA cancellation performance even for clutter echoes coming from the front lobe region after a preliminary DSI suppression.

In order to investigate the effect of an angle-dependent channel imbalance on the clutter cancellation performance of the DPCA scheme, we consider a simulated clutter scenario for a moving passive radar. This also removes any other factor potentially affecting the experimental data.

Clutter returns are generated according to (1), for a scene spanning $N_{R}=250$ range cells. Amplitudes $A_{q}(\varphi)$ associated with different clutter patches are assumed independent and identically distributed complex gaussian variables, resulting in a homogeneous clutter scenario. Omnidirectional antennas are considered, within an angular sector $\varphi=[0, \pi]$ (no back-lobe contributions). A DVB-T sequence is used as reference signal. Geometry, signal and system parameters are selected to match those of the experimental setup (see Table I). The generated input signal includes clutter returns and thermal noise with an input clutterto-noise-ratio (CNR) of $20 \mathrm{~dB}$; to this purpose, the overall clutter contribution is scaled to have a power level of $20 \mathrm{~dB}$ above noise level (deliberately set to unity), at the input of each channel. Absence of ICM is assumed and an angle dependent imbalance $\Gamma(\varphi)$ between the two receiving channels is included. Specifically, we assume a sinusoidal phase imbalance, both in azimuth and elevation angle, which maps onto the Doppler frequency axis as illustrated in Fig. 11(a).

The range-Doppler map obtained from a single channel is reported in Fig. 11(b). The corresponding range-Doppler map at the output of the DPCA scheme is shown in Fig. 11(c), when a SCC strategy is applied for channel calibration. As 


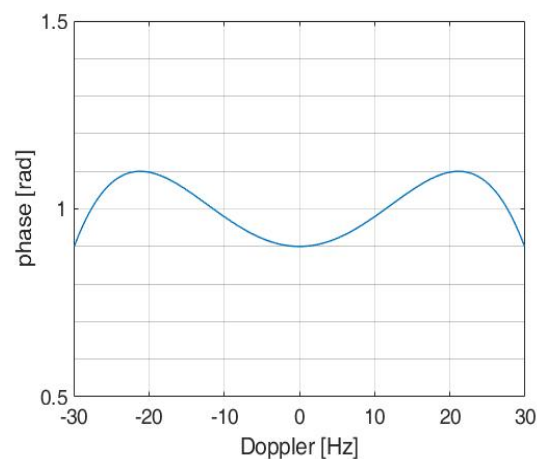

(a)

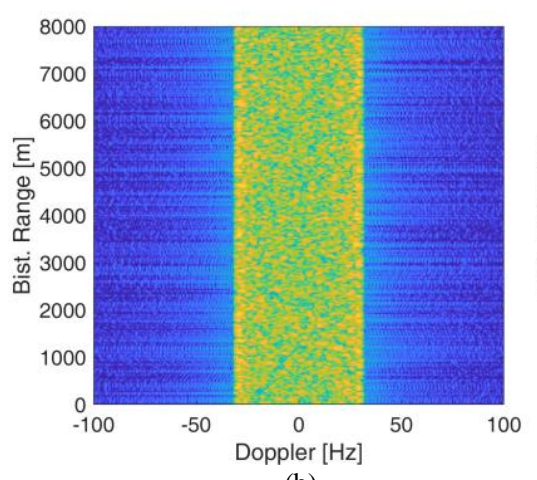

(b)

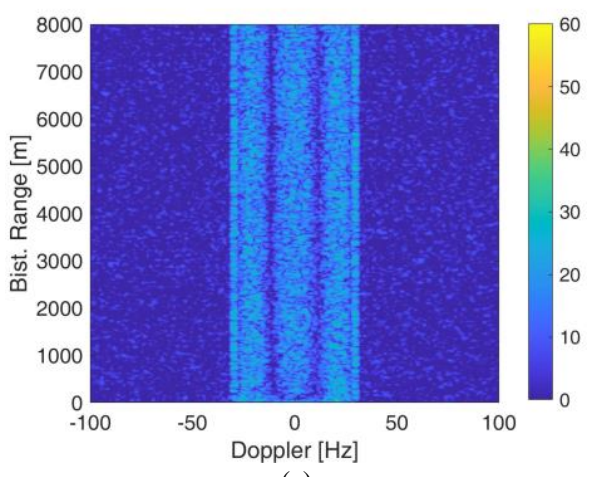

(c)

Fig. 11. Effect of angle dependent channel imbalance in simulated clutter scenario: (a) simulated phase imbalance as a function of Doppler frequency; (b) single channel range-Doppler map; (c) range-Doppler map after DPCA with SCC approach.

expected, only the average component of the imbalance is corrected and an imperfect clutter cancellation is achieved, due to the uncompensated imbalance fluctuation. Therefore, a Doppler-dependent clutter cancellation performance is obtained, being the highest cancellation achieved where the phase imbalance is close to its average value.

In order to compensate for these effects and further improve clutter attenuation performance, more refined clutter-based channel calibration strategies are introduced and compared below. All the considered strategies rely on the two-stage cancellation scheme presented in Section IV, but they overcome the simplified approach based on a single calibration coefficient by looking for a set of coefficients to be applied across one dimension or two dimensions, namely Doppler or range/Doppler.

\section{A. Doppler dependent calibration (DDC)}

We start from the model in (4) and we consider the potential variation of channel imbalance as a function of the angle of arrival, i.e. $G_{q}^{(T A)}(\varphi) / G_{q}^{(L A)}(\varphi)=\Gamma(\varphi)$. We also assume that clutter returns at a generic Doppler bin $m$ are dominated by the response of the scatterers belonging to the angular sector defined by the Doppler resolution, while contributions from different directions are negligible. This assumption holds when an appropriate windowing is adopted at the Doppler processing stage, which yields low sidelobes for the $g_{d}[m]$ function in (4). For the sake of clarity, the angular sector is centred at $\varphi_{m}$ so that $\frac{v_{p}}{\lambda} \cos \varphi_{m}=m \delta f$ and its width is given by $\delta \varphi_{m} \cong \frac{\lambda}{v_{p} \sin \varphi_{m}} \delta f, \delta f$ being the Doppler resolution.

For sufficiently high Doppler resolution (narrow angular sector) and/or sufficiently slow variation of $\Gamma(\varphi)$, the clutter contributions at the output of the two range-Doppler maps can be expressed as:

$$
\begin{aligned}
& z_{C}^{(L A)}[l, m]=\sum_{q=1}^{N_{R}} G_{q}^{(L A)}\left(\varphi_{m}\right) A_{q}\left(\varphi_{m}\right) g_{r}\left[l-l_{\tau_{q}}\right] g_{d}[0] \delta \varphi_{m} \\
& z_{C}^{(T A)}[l, m]=\sum_{q=1}^{N_{R}} G_{q}^{(T A)}\left(\varphi_{m}\right) A_{q}\left(\varphi_{m}\right) g_{r}\left[l-l_{\tau_{q}}\right] g_{d}[0] \delta \varphi_{m}
\end{aligned}
$$

As a result, for clutter signal the following relation holds: $z_{c}^{(T A)}[l, m] \cong \Gamma\left(\varphi_{m}\right) \cdot z_{c}^{(L A)}[l, m]$.

This assumption allows us to exploit the one-to-one relationship between angle of arrival and Doppler frequency of stationary scatterers in order to provide an angledependent compensation of channel imbalance. Specifically, we propose a Doppler dependent calibration (DDC) strategy, where we look for complex calibration coefficients to be separately applied at each Doppler bin so that the output clutter power after DPCA subtraction is minimized at that Doppler bin.

By proceeding as in (7)-(8), the correction coefficient at the $m$-th Doppler bin can be estimated as:

$$
\hat{\Gamma}[m]=\frac{\sum_{l=l_{1}}^{l_{2}} z^{(T A)}[l, m] z^{(L A)^{*}}[l, m]}{\sum_{l=l_{1}}^{l_{2}}\left|z^{(L A)}[l, m]\right|^{2}}
$$

where the average values at both the numerator and denominator are evaluated over consecutive range cells spanning indexes from $l_{1}$ to $l_{2}$.

This approach assumes that scatterers belonging to the same Doppler bin, and hence to the same angular direction, are characterized by analogous amplitude and phase channel imbalance, namely a nearly uniform imbalance in range.

It is worth noting that such Doppler dependent calibration technique is able to compensate not only for angle-dependent antenna pattern mismatch, but it also intrinsically compensates for any phase slope in Doppler, resulting from residual channel displacement, thus making unnecessary the fine delay compensation step in Fig. 7 and adaptively establishing the DPCA condition.

A similar methodology for digital channel balancing was proposed in [33]-[34], in the context of multi-channel SARGMTI applications, where an iterative algorithm is operated in the 2-D frequency domain to calibrate spectral response of different channels. In our case, the relatively small bandwidth of exploited waveform, compared to typical SAR signals, allows to assume negligible difference in channel frequency response and to operate calibration only in the Doppler domain. Clearly, such data driven calibration strategies based on clutter echoes are more effective when high values of CNR are present, which is the case of our experiment. 
(C) 2020 IEEE. Personal use of this material is permitted. Permission from IEEE must be obtained for all other uses, in any current or future media, including reprinting/republishing this material for advertising or promotional purposes, creating new collective works, for resale or redistribution to servers or lists, or reuse of any copyrighted component of this work in other works. DOI: 10.1109/TAES.2020.2987478.

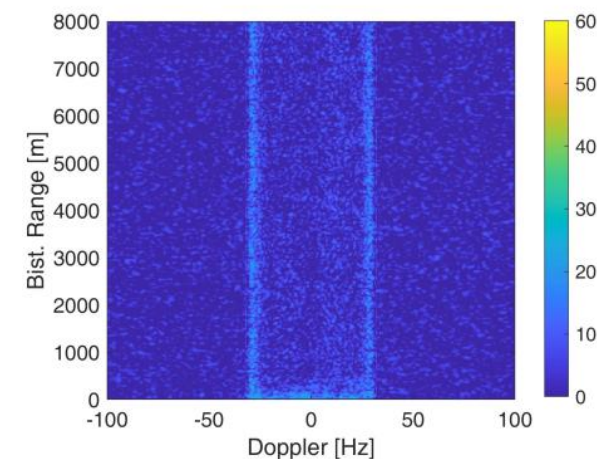

(a)

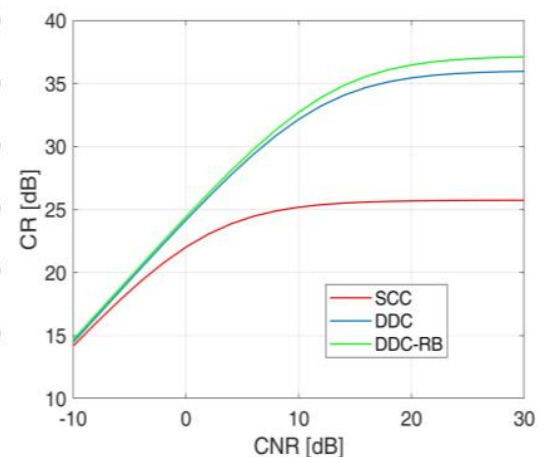

(b)

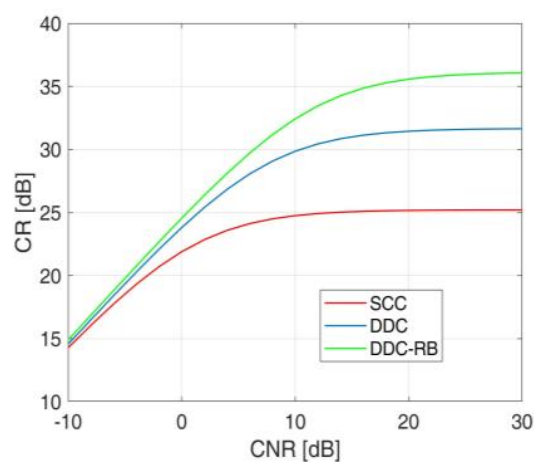

(c)

Fig. 12. Analysis of adaptive clutter-based calibration techniques against simulated clutter scenario in the presence of angle dependent channel imbalance: (a) range-Doppler map after DPCA with DDC approach; (b) comparison of average CR obtained as a function of input CNR; (c) comparison of average $\mathrm{CR}$ in a different geometry with increased receiver altitude.

The idea of performing an angle/Doppler dependent calibration for multi-channel passive radar data has been firstly presented in [12], based on a minimum variance power spectrum estimation. However, the approach adopted in [12] only allows angle-dependent phase imbalances to be estimated and compensated for, which is not effective in the presence of significant amplitude imbalance, as demonstrated in the following.

Fig. 12(a) shows the range-Doppler map obtained by applying the DPCA scheme combined with a DDC approach, for the same simulated clutter case of Fig. 11. As expected, a significant improvement in terms of clutter cancellation capability is achieved, compared to the SCC case, thanks to the possibility to compensate also for imbalance fluctuations. Notice that the effectiveness of the channel calibration, and thus the cancellation performance, decreases when approaching the edges of clutter Doppler spectrum. This effect can be traced back to limited validity of the approximation made in (9). In fact, due to the non-linear relation between Doppler frequency and angle of arrival, in those regions Doppler resolution maps broader angular sectors, which may correspond to different values of channel imbalance, thus reducing the ability to compensate for imbalance variations.

In Fig. 12(b) we show the average CR obtained, as a function of input CNR, for the same simulated clutter scenario. We notice that the CR initially improves as the input CNR increases, being the cancellation ideally limited only by thermal noise. However, when further increasing the CNR, the presence of uncancelled clutter residuals leads to a saturation of the cancellation performance. As expected, the DDC approach allows to achieve significantly higher values of cancellation, compared to the SCC approach, thanks to the adaptation capability in the Doppler dimension which compensates for imbalance variations.

Fig. 13 and Fig. 14, respectively show the range-Doppler map after DPCA subtraction and the corresponding CR map, resulting from the application of the DDC approach in (10) to the experimental data set under consideration. Application is limited to Doppler frequency bins within the clutter bandwidth [-32;32] Hz and bistatic range interval [150;8000] $\mathrm{m}$. The CR reported in Fig. 14 takes into account the DPCA stage only, assuming that this is applied after the removal of the DSI by means of the ECA approach. By comparing these results with those in Fig. 8 and Fig. 10, a substantial improvement in clutter cancellation performance can be observed, with $\mathrm{CR}$ values increased by more than $10 \mathrm{~dB}$ at specific locations.

The amplitude and phase of the estimated calibration coefficients are shown in Fig. 15 as a function of Doppler frequency (respectively solid blue and red lines). Their

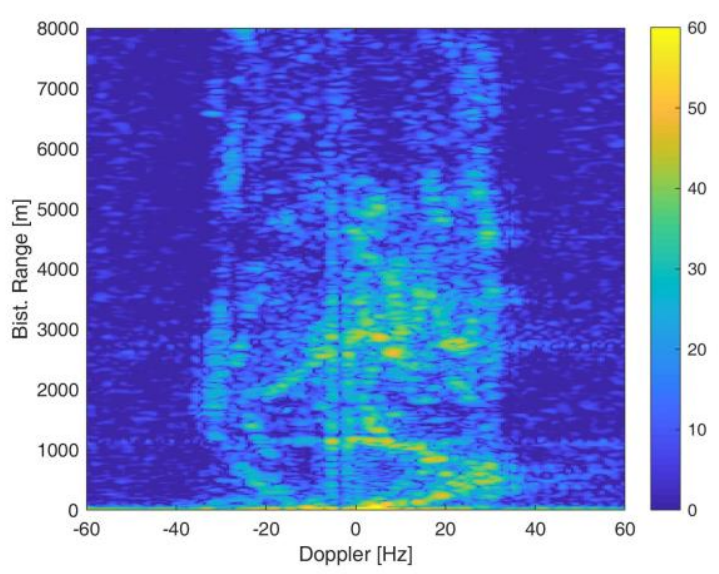

Fig. 13. Range-Doppler map after two-stage cancellation scheme with DDC approach.

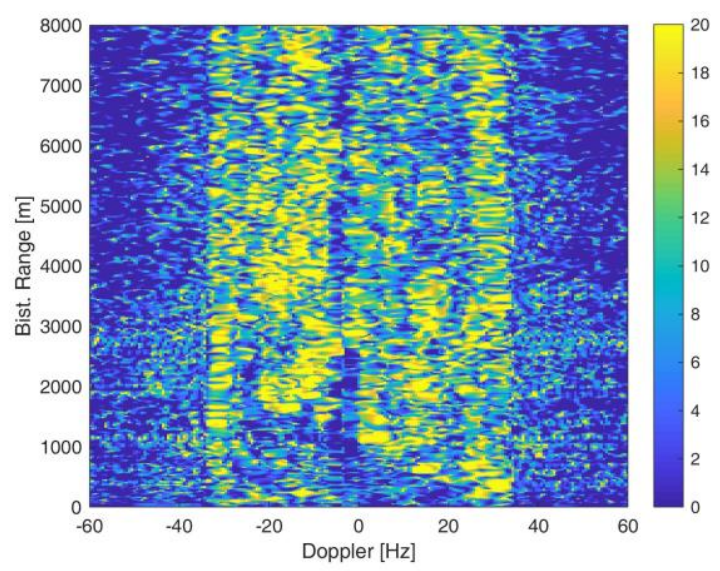

Fig. 14. Cancellation Ratio obtained after the DPCA stage with DDC approach. 
average values are consistent with the amplitude and phase imbalance estimated by means of the SCC strategy (reported in figure by dashed horizontal lines). However, a sensible variation of channel imbalance across Doppler frequencies can be noticed both in amplitude and in phase. Moreover, a slight phase slope is apparent, possibly due to a non-perfect delay compensation between LA and TA channels.

In order to assert with more confidence that the proposed digital calibration strategy is providing a reliable estimate of the actual angle-dependent channel imbalances, we include in Fig. 15 the corresponding curves obtained by averaging (10) over an acquisition time of 10 seconds (see dotted lines). Specifically, the average amplitude and phase calibration coefficients are evaluated based on subsequent nonoverlapped CPIs, where the platform was verified to maintain constant motion parameters. The dotted curves clearly resemble the estimates obtained at the CPI under consideration, thus demonstrating that a reliable and stable information is extracted with the proposed approach

As is apparent, the highest improvement in terms of CR between Fig. 14 and Fig. 10 is obtained at Doppler values where the largest deviations are observed in the estimated calibration coefficients (solid lines) with respect to the correction suggested by the SCC approach (dashed lines). This is the case of the Doppler region at about $-30 \mathrm{~Hz}$, where the enhanced clutter cancellation obtained after the DDC approach now yields an easier discrimination of the cooperative target Delphin, approximately at bistatic range $6600 \mathrm{~m}$ (see Section VI.B for more details).

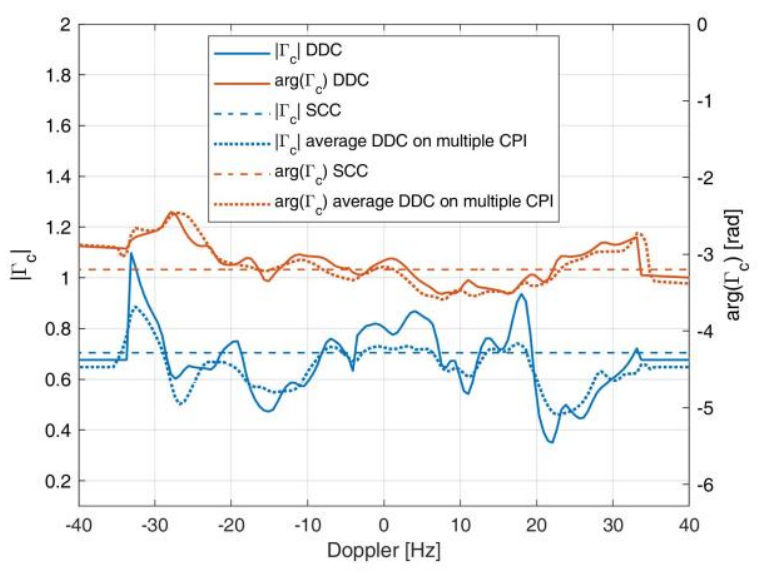

Fig. 15. Amplitude and phase of the estimated calibration coefficients as a function of Doppler frequency: DDC on current CPI (solid line); DDC averaged over multiple CPIs (dotted lines); values from SCC (dashed lines).

\section{B. Doppler dependent calibration in range bands $(D D C$ - $R B)$}

Further performance improvement can be achieved by making the calibration technique adaptive also in the range dimension. The idea is to compensate for potential additional channel mismatch as a function of range, as this is largely related to antenna pattern differences in elevation.

In the considered experiment, this additional degree of freedom in the calibration process is not expected to give significant improvement, since a ground-based receiver experiences small variation in the elevation angle of observed clutter, mostly associated to changes in terrain height. Moreover, a relatively small extension in range has been considered in the available data files. On the other hand, it is reasonably expected to be more beneficial for bistatic configurations exploiting an airborne receiver.

Adaptation capability in range can also be useful to mitigate the effects of clutter contributions coming from the antenna back-lobe, which typically affect the first range bins and might set different constraints on the calibration coefficients.

Starting from the simplified model in (9), we take into account also the variation of imbalance in range, in addition to the variation in angle, i.e. $G_{q}^{(T A)}(\varphi) / G_{q}^{(L A)}(\varphi)=\Gamma_{q}(\varphi)$. By proceeding as in section V.A, we assume that clutter returns at a generic range-Doppler bin $[l, m]$ are dominated by the response of the scatterers belonging to the clutter patch defined by the range and Doppler resolution. To guarantee the validity of this assumption, an appropriate windowing is adopted to achieve low sidelobes both in range and in Doppler, namely for both $g_{d}[m]$ and $g_{r}[l]$ functions in (4). Therefore, for clutter signal at the output of the LA and TA range-Doppler maps, the following relation holds: $z_{c}^{(T A)}[l, m] \cong \Gamma_{q_{l}}\left(\varphi_{m}\right) \cdot z_{c}^{(L A)}[l, m]$.

This assumption inherently requires to compensate also for the range-dependent channel imbalance before applying DPCA stage. The simplest approach along this line, assuming a relatively slow variation of imbalance with range, is to subdivide the clutter area of interest in range bands and to apply a Doppler dependent calibration, according to (10), at each range band separately (DDC-RB).

In Fig. 12(b) the SCC, DDC and DDC-RB approaches are compared in terms of average CR obtained for the simulated clutter scenario, as a function of the input CNR. We notice that the DDC-RB approach, applied by subdividing the clutter area into four non-overlapped range bands of $2000 \mathrm{~m}$ each, achieves the highest values of cancellation, thanks to the resulting adaptation capability in both Doppler and range dimensions. As expected, the improvement with respect to DDC approach is limited, since the effect of the imbalance variation in elevation is limited to the first few range bins due to the considered observation geometry (ground-based receiver). In Fig. 12(c) a different geometry is considered, where assumed receiver altitude is increased from $3 \mathrm{~m}$ to 100 $\mathrm{m}$. In this case the effect of imbalance variation in elevation is more significant and allows to better appreciate the advantages of the DDC-RB approach compared to the DDC.

In order to assess the achievable improvements in terms of cancellation performance and compare the different calibration techniques against the experimental data set, in Fig. 16 we consider the curves of achieved CR as a function of Doppler frequency, which express the average power attenuation provided at each Doppler bin by the DPCA stage. Specifically, this is defined as in (6) where the power at the input and at the output of the DPCA stage at a given Doppler bin has been averaged over the available range gates.

Notice that values of CR also depend on the input clutterto-noise ratio across Doppler bins, being the highest values expected at the Doppler bins where the strongest clutter contributions appear. Besides this consideration, here we focus on the comparative analysis of the results obtained 
adopting the SCC, DDC and DDC-RB approaches for channel calibration.

As expected from previous section, an appreciable improvement is achieved by applying a Doppler dependent calibration (blue dash-dot curve), compared to a single coefficient approach (red dashed curve). Apparently, the CR can then be further increased by few $\mathrm{dBs}$, if calibration is applied in range bands (green solid curve). Specifically, in this case, the region of interest in bistatic range has been subdivided into five non-overlapped bands of $1600 \mathrm{~m}$ each Obviously, a trade-off exists in selection of range bands dimension. On one hand, a smaller extension in range would result in a better cancellation performance; on the other hand, it might cause calibration process to be influenced by the presence of outliers, there including potential targets contributions.

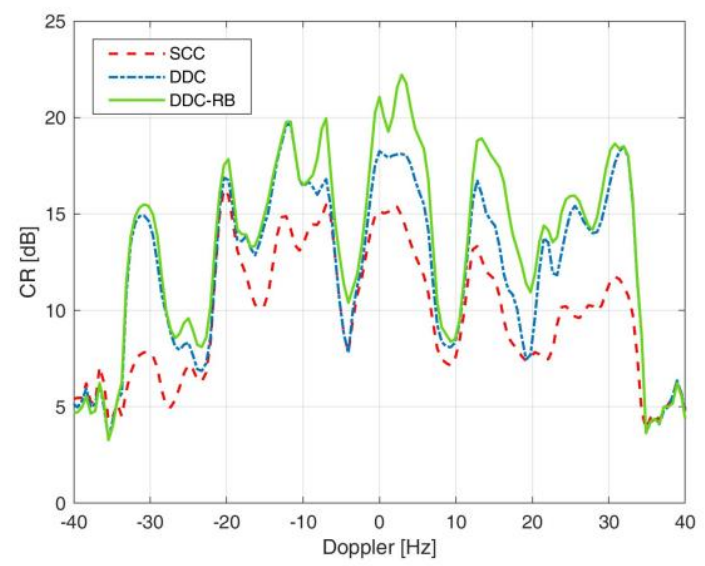

Fig. 16. Comparison of average Cancellation Ratio given by the DPCA stage as a function of Doppler frequency for different calibration techniques.

\section{Robust DDC-RB}

When exploiting a Doppler dependent calibration strategy applied across range bands, the support (i.e. the number of bins in the range-Doppler map) used to estimate the required coefficients is deliberately reduced in order to track changes of the channel imbalances over the considered area.

However, in such condition, the effectiveness of the proposed strategy could be jeopardized by the presence of potential outliers as their effect does not average out in the summation in (10). Such outliers might include:

- range-Doppler bins that are severely corrupted by noise,

- strong echoes from stationary scatterers impinging on the antenna back-lobes and affected by a different channel imbalance with respect to the scatterers lying in the area under consideration,

- sidelobes associated to strong scatterers belonging to a different area, as well as

- potential strong moving targets in super clutter visibility condition.

If enough range bins are available for the estimation of a Doppler dependent channel imbalance, it is reasonable to assume that the presence of outliers has a negligible impact. Conversely, if few range bins are used, calibration becomes more sensitive to potential outlying values, preventing an effective clutter cancellation. In addition, targets with high signal to clutter ratio (SCR) may result in being partially suppressed.

In order to avoid this undesirable effect and improve robustness of the proposed adaptive calibration technique, we start from the following consideration. Clutter contributions belonging to same Doppler bin, and hence to same angular direction, within an appropriate extension in range, are presumably characterized by similar channel imbalance. Therefore, the values extracted at the corresponding bins, tend to be concentrated around the true local value, apart for an intrinsic fluctuation that depends on the local CNR. Specifically, we observed that the phase component of the sample estimates is typically quite stable across range cells. Therefore, when estimating the calibration coefficient, potential strong outliers could be in principle excluded based on their interferometric phase values.

The aim is to achieve a channel calibration ideally based on clutter contributions only and to avoid the influence of potential outliers. To this purpose, the calibration coefficient is evaluated for each Doppler frequency, according to (10), by exploiting only range bins whose phase difference between LA and TA channels does not deviate by more than an assigned threshold $\eta$ from a median value estimated on that specific Doppler frequency:

$$
\begin{gathered}
\hat{\Gamma}[m]=\frac{\sum_{l \in \Lambda_{\mathrm{m}}} Z^{(T A)}[l, m] Z^{(L A)^{*}}[l, m]}{\sum_{l \in \Lambda_{\mathrm{m}}}\left|Z^{(L A)}[l, m]\right|^{2}} \\
\Lambda_{m}=\{l:|\Delta \phi[l, m]-\operatorname{median}(\Delta \phi[l, m])|<\eta\} \quad \forall m
\end{gathered}
$$

where $\Delta \phi$ indicates the phase difference between coregistered channel maps:

$$
\Delta \phi[l, m]=\arg \left\{z^{(T A)}[l, m] z^{(L A)^{*}}[l, m]\right\}
$$

Median value is preferred to average value, since it is less sensitive to outliers. Moreover, to make estimation more robust, only the first $\mathrm{M}$ bins with higher power level are considered. In this way, by selecting a proper threshold value, potential strong moving targets, as well as echoes heavily corrupted by noise, can be excluded from the calibration process, thus improving its robustness.

To prove the effectiveness of the proposed solution, Fig. 17 shows the results of channel calibration and DPCA on the cooperative aerial target. In particular, Fig. 17(a) shows an enlarged view of a single channel range-Doppler map around the target position. Fig. 17(b) and Fig. 17(c) show the resulting maps after channel calibration and DPCA subtraction, respectively without and with the application of phase outliers exclusion technique in (11). In the latter case, the threshold $\eta$ is empirically set to $1 \mathrm{rad}$. DDC-RB approach is applied using narrow range bands of $800 \mathrm{~m}$, in order to stress the effect of the presence of outliers.

A significant clutter attenuation is achieved in both cases, allowing the target echo to be easily detected. The estimated SCNR is increased by more than $10 \mathrm{~dB}$ in both Fig. 17(b) and 


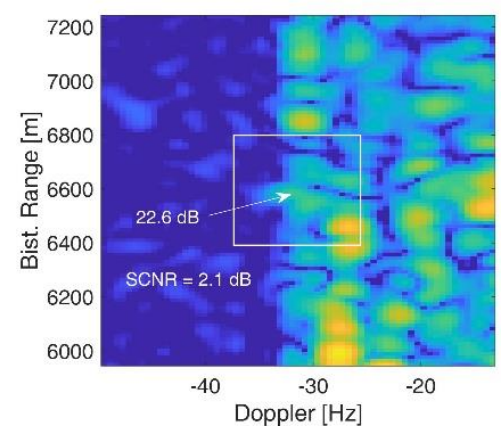

(a)

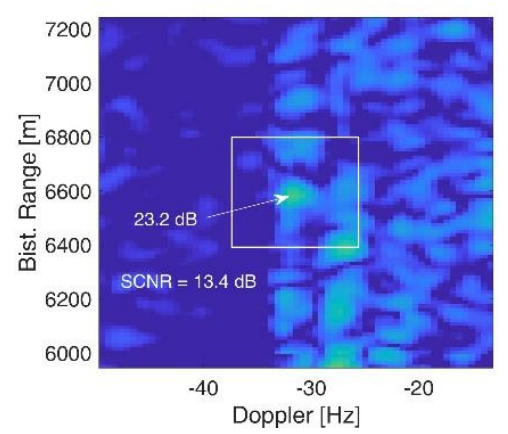

(b)

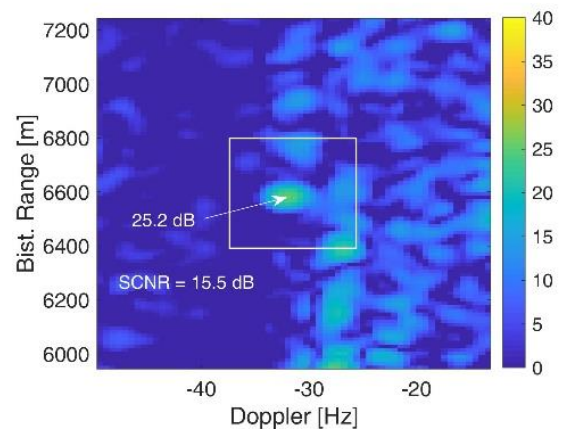

(c)

Fig. 17. Results of cancellation scheme on real aerial target: (a) before DPCA subtraction ; (b) after DPCA subtraction with DDC-RB; (c) after DPCA subtraction with Robust DDC-RB excluding outliers. Narrow range bands of $800 \mathrm{~m}$ are used for calibration.

Fig. 17(c) compared to Fig. 17(a). However, we observe that the influence of moving target on calibration, appreciable in Fig. 17(b), decreases the gain on target and the clutter cancellation capability on the corresponding Doppler bins. These effects are effectively mitigated in Fig. 17(c) thanks to the proposed approach. This results in a further increase in terms of SCNR by $2.1 \mathrm{~dB}$.

Notice that the exclusion of outliers can be applied also to the DDC approach, despite it is expected to be less sensitive to the presence of outliers. In the following, this will be referred to as Robust DDC. Benefits of excluding outliers from the calibration process will be further investigated in the next section, where the effectiveness of the considered processing scheme and the proposed calibration strategies is analysed in terms of clutter suppression as well as moving target detection capability.

\section{Computational effort}

For the reader's convenience, the proposed channel calibration algorithms are summarized in Table II, listing the adopted acronyms and their main characteristics. In addition, a comparison of the required computational effort is also included, in terms of number of complex multiplications needed for the estimation of the corresponding calibration coefficients. In fact, the cost required for the application of calibration to one of the channels is the same for all techniques. $\mathrm{N}_{\mathrm{d}}$ and $\mathrm{N}_{\mathrm{r}}$ denote respectively the number of
Doppler and range bins considered for calibration, corresponding to the selected clutter area of interest; $\mathrm{n}_{\mathrm{r}}$ is the number of range bands in which the area is subdivided. Apart from DSI-based calibration (where a single complex multiplication is required), the other clutter-based techniques, namely SCC, DDC and DDC-RB, have a comparable computational effort, which depends on the range and Doppler extension of the considered clutter area. Notice that Robust versions of algorithms need an additional cost, indicated as a function of the number of range bins, due to operations required for exclusion of outliers. This additional cost cannot be easily expressed in terms of number of multiplications, since it requires sorting operations, and in any case it does not constitute a dominant contribution to the overall computational cost. Moreover, it is worth noting that the computational effort required by considered calibration approaches is substantially lower than that required for the evaluation of the range-Doppler maps.

\section{PERFORMANCE ANALYSIS AGAINST TARGETS}

In this section, the performance of the considered processing scheme is investigated against real and synthetic moving targets. The effectiveness of the DPCA approach in suppressing clutter returns, while preserving moving target echoes, is verified when applying the different calibration strategies introduced in the previous sections. The results are

TABLE II. SUMMARY OF CONSIDERED CHANNEL CALIBRATION TECHNIQUES

\begin{tabular}{|c|c|c|c|}
\hline Technique & Reference & Description & Number of complex multiplications \\
\hline DSI-based & Section III.C, eq. (5) & $\begin{array}{c}\text { Estimation of single calibration } \\
\text { coefficient based on DSI }\end{array}$ & 1 \\
\hline $\begin{array}{c}\text { SCC (single calibration } \\
\text { coefficient) }\end{array}$ & Section IV, eq. (8) & $\begin{array}{c}\text { Estimation of single calibration } \\
\text { coefficient based on clutter area }\end{array}$ & $2 \mathrm{~N}_{\mathrm{d}} \mathrm{N}_{\mathrm{r}}+1$ \\
\hline $\begin{array}{c}\text { DDC (Doppler dependent } \\
\text { calibration) }\end{array}$ & Section V.A, eq. (10) & $\begin{array}{c}\text { Estimation of } \mathrm{N}_{\mathrm{d}} \text { coefficients as a } \\
\text { function of Doppler frequency }\end{array}$ & $\mathrm{N}_{\mathrm{d}}\left(2 \mathrm{~N}_{\mathrm{r}}+1\right)$ \\
\hline $\begin{array}{c}\text { DDC-RB (Doppler dependent } \\
\text { calibration in range bands) }\end{array}$ & Section V.B & $\begin{array}{c}\text { Estimation of } \mathrm{N}_{\mathrm{d}} \mathrm{x} \mathrm{n}_{\mathrm{r}} \text { coefficients as a } \\
\text { function of Doppler frequency and } \\
\text { range band }\end{array}$ & $\mathrm{N}_{\mathrm{d}}\left(2 \mathrm{~N}_{\mathrm{r}}+\mathrm{n}_{\mathrm{r}}\right)$ \\
\hline Robust DDC & Section V.C, eq. (11) & $\begin{array}{c}\text { Robust version DDC to avoid the } \\
\text { influence of strong outliers }\end{array}$ & $\mathrm{N}_{\mathrm{d}}\left(2 \mathrm{~N}_{\mathrm{r}}+1\right)+\mathrm{N}_{\mathrm{d}} f\left(\mathrm{~N}_{\mathrm{r}}\right)$ \\
\hline Robust DDC-RB & Section V.C & $\begin{array}{c}\text { Robust version of DDC-RB to avoid } \\
\text { the influence of strong outliers }\end{array}$ & $\mathrm{N}_{\mathrm{d}}\left(2 \mathrm{~N}_{\mathrm{r}}+\mathrm{n}_{\mathrm{r}}\right)+\mathrm{N}_{\mathrm{d}} \mathrm{n}_{\mathrm{r}} f\left(\mathrm{~N}_{\mathrm{r}} / \mathrm{n}_{\mathrm{r}}\right)$ \\
\hline
\end{tabular}


compared in terms of achievable improvement in signal-todisturbance ratio.

A single cooperative aerial target was present in the experimental campaign and, due to its relatively high speed compared to the ground moving platform, it is rarely found within the clutter Doppler spectrum. Therefore, additional simulated moving targets are injected into real data, in order to have more chances for validating the proposed techniques.

\section{A. Target model and effectiveness of proposed approach}

A synthetic target signal is generated from the reconstructed reference signal by applying specific delay and Doppler shift according to desired target bistatic range $R_{b}$ and bistatic radial velocity $v_{b}$, according to the following model:

$$
\begin{array}{r}
r_{0}^{(L A)}[l] \cong A_{0} \sum_{n} s_{n}\left[l-n L-l_{\tau_{0}}\right] e^{j 2 \pi f_{D_{0}} n T} \\
r_{0}^{(T A)}[l] \cong \Gamma_{C}\left(\varphi_{0}\right) A_{0} \sum_{n} s_{n}\left[l-n L-l_{\tau_{0}}\right] \\
\cdot e^{j 2 \pi f_{D_{0}} n T} e^{-j 2 \pi \frac{d}{\lambda} \cos \varphi_{0}}
\end{array}
$$

where $A_{0}$ is the target complex amplitude, $l_{\tau_{0}}=f_{s} R_{b} / c$ is the bistatic propagation delay ( $f_{s}$ being the sampling frequency), $\varphi_{0}$ is the angle between platform velocity vector and receiver to target line of sight and $f_{D_{0}}$ is the target bistatic Doppler frequency given by:

$$
f_{D_{0}}=\frac{v_{p}}{\lambda} \cos \varphi_{0}-\frac{v_{b}}{\lambda}
$$

Proper amplitude and phase imbalance coefficient $\Gamma_{c}\left(\varphi_{0}\right)$ is also applied to the generated target echoes between LA and TA channels, according to the imbalance estimated on clutter at the same angular direction, in the absence of targets.

The SCNR at the output of the DPCA stage is used in the following to compare the performance of different strategies. By definition, the SCNR improvement factor (IF) is equivalent to the product of disturbance $\mathrm{CR}$ and target gain $(\mathrm{G})$, i.e. $I F=C R \cdot G$. Notice that, in this case, the achievable target gain is limited to a maximum value of $3 \mathrm{~dB}$.

Specifically, for the case of injected targets, the target gain can be easily measured by comparing the power level of the input and output maps at the target range-Doppler location, when the processing scheme is fed with target echoes only. Correspondingly, the disturbance power level is estimated over a proper area surrounding the target location, by exploiting the maps containing just clutter and noise at the input and output of the DPCA stage. However, in both cases, the proposed channel calibration techniques are applied against data including both target echoes and disturbance contributions, in order to take into account the effects of targets on the calibration process.

In order to extensively investigate the effectiveness of the proposed calibration techniques, we focus on a single synthetic target, T1, whose parameters are listed in Table III.
Results are reported in terms of disturbance cancellation ratio (Fig. 18(a)) and output SCNR (Fig. 18(b)), achieved after DPCA subtraction, as a function of the input SCNR used to generate the synthetic target echoes. Specifically, we compare the DPCA performance obtained after the application of the DSI-based calibration, the SCC, the DDC, and the DDC-RB. Also, the robust versions of the latter two approaches, namely the Robust DDC and the Robust DDC$\mathrm{RB}$, are reported in order to understand the benefits of the outliers exclusion strategy.

As is apparent, when exploiting a DSI-based calibration strategy, a quite low CR value is obtained (see the grey curve in Fig. 18(a)). This is well in line with the results in Fig. 5 and Fig. 6 and, in turn, results in a limited SCNR gain between the input and the output of the cancellation stage (see Fig. 18(b)) so that, for instance, an input SCNR of $10 \mathrm{~dB}$ is required for the output SCNR to exceed $15 \mathrm{~dB}$.

Despite still exploiting a single adaptive coefficient for the channel calibration, the SCC allows to significantly increase the $\mathrm{CR}$ and the subsequent SCNR gain with respect to the DSI-based approach. Moreover, thanks to the average performed across a wide region of the range-Doppler map, the SCC is not affected by the presence of the target up to very high values of the input SCNR. Therefore, the SCC could be regarded as a simple and effective approach in practical applications

However, the use of more sophisticated adaptive calibration strategies, compensating for Doppler and range dependent channel imbalance, allows to further improve the cancellation performance and, consequently, the SCNR gain. In particular, when exploiting the DDC or the DDC-RB (operating over range bands of $1600 \mathrm{~m}$ ), the CR improves by $2 \mathrm{~dB}$ and $3 \mathrm{~dB}$, respectively, compared to the SSC. Accordingly, in the considered case study, a remarkable input/output SCNR gain (slightly higher than $20 \mathrm{~dB}$ ) is achieved with the DPCA applied after the DDC-RB, at least when the input SCNR is reasonably low.

As expected, localized adaptive calibration approaches are more sensitive to the presence of targets with high input SCNR values, as they represent outliers in the estimation of the calibration coefficients. This has non-negligible effects on both clutter cancellation performance, due to a corrupted channel imbalance estimation, and the resulting output SCNR, since this effect might yield a partial suppression of target signal. In fact, in the considered case study, both the DDC and the DDC-RB experience significant degradations as the input SCNR increase (see dashed lines in Fig. 18(a-b)). Incidentally, we observe that this effect appears at lower SCNR values when exploiting the DDC-RB, since fewer range bins are used for estimating the calibration coefficients. In contrast, the DDC benefits from the average performed across a wider range extent so that the effect of outliers becomes apparent at higher SCNR values.

This undesirable effect can be avoided by making the calibration process robust against outliers, according to the strategy described in Section V.C. Both the Robust DDC and the Robust DDC-RB allow to recover the CR loss due to the target influence on the calibration stage (see solid lines in Fig. 18(a)). Accordingly, the moving target echoes are correctly preserved and a remarkably high SCNR gain is restored even 


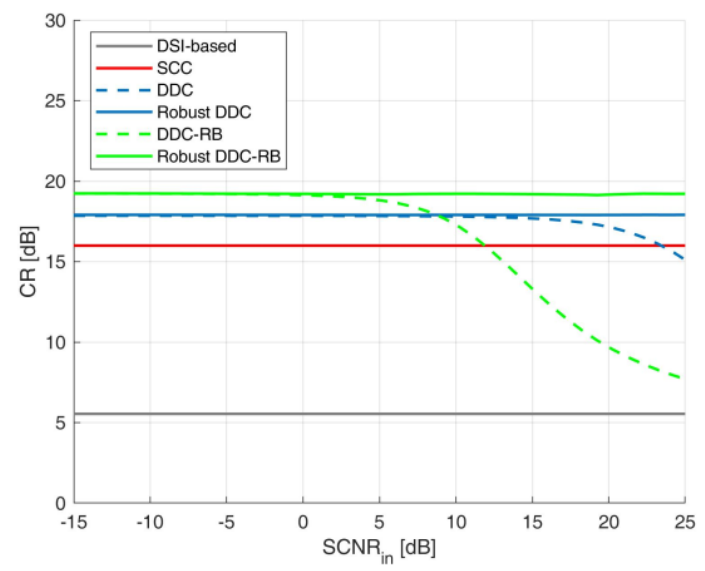

(a)

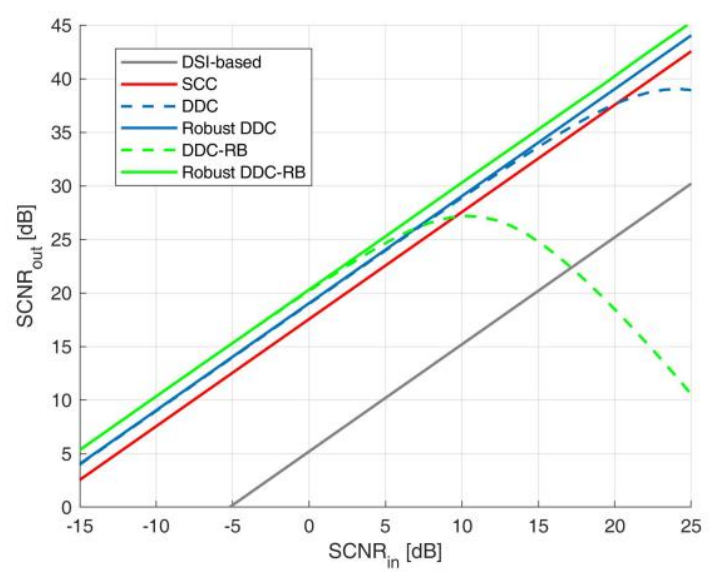

(b)

Fig. 18. Performance analysis of DPCA adopting different channel calibration strategies, as a function of target SCNR in input: (a) disturbance Cancellation Ratio; (b) achieved target SCNR in output.

when the target power level is several $\mathrm{dBs}$ above the disturbance background at the input of the DPCA. Nevertheless, it is worth noting that such strong targets might be detected even before clutter suppression or by means of a less refined calibration strategy.

In order to understand the effect of the adopted range band size, we report in Fig. 19 the performance of the DPCA stage after the DDC-RB and the Robust DDC-RB as a function of the dimension of considered range band. In this case the input SCNR is set to a constant arbitrary value of 5 $\mathrm{dB}$. As expected, the two approaches provide largely comparable results for large enough range bands. In contrast, the performance of the DDC-RB progressively degrades as the range band size decreases. When using the Robust DDC$\mathrm{RB}$, a limited reduction of range band extension can improve the cancellation ratio with respect to the results in Fig. 18, thus providing a higher SCNR at the output of the DPCA stage. Clearly, beyond a certain limit, identification of outliers is no more feasible, and the performance rapidly degrades. This analysis also shows that the choice of range bands of $1600 \mathrm{~m}$ adopted so far allows a good trade-off between performance and robustness to outliers in the case under consideration. This value will be also used for the analyses reported in the next section.

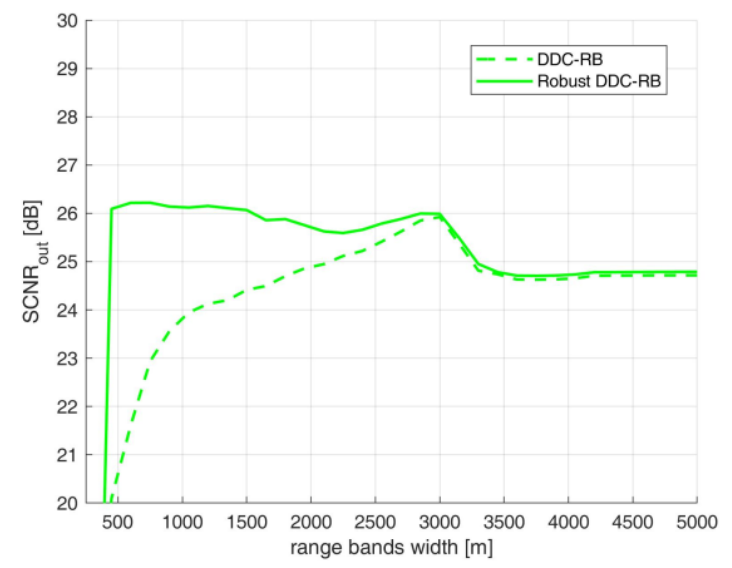

Fig. 19. Performance analysis of DPCA adopting DDC-RB and Robust DDC-RB, as a function of range bands size: achieved target SCNR in output.

\section{B. Performance comparison}

It is expected that the performance of the proposed approaches might vary across the range-Doppler map depending on the local clutter characteristics. Therefore, in order to further investigate the effectiveness of the considered strategies and to compare the achievable performance, results are reported below against multiple injected targets, as well as against the real cooperative target. Parameters of real and simulated targets are listed in Table III. Different angles of arrival are considered for injected targets and bistatic velocity values are set so that they do not coincide with the expected blind velocities $p \lambda / T_{D P C A}(p \in \mathbb{Z})$.

The range-Doppler maps before and after DPCA subtraction are shown in Fig. 20(a-b). Specifically, the Robust DDC-RB approach is adopted for channel calibration. A significant reduction of clutter power is achieved, so that both real and simulated targets are clearly visible in the final map (see Fig. 20(b)) and they could be easily detected by a conventional CFAR scheme.

Results in terms of disturbance cancellation ratio (CR), target gain $(\mathrm{G})$ and corresponding SCNR improvement factor (IF), obtained for the different channel calibration strategies are reported in Table IV. As expected, a significant gap in DPCA performance is present between the DSI-based calibration and the other clutter-based calibration techniques.

Moreover, all targets, in different measure, benefit from the enhanced clutter cancellation capability given by the Robust DDC approach, compared to the SCC. In addition, the Robust DDC-RB allows a further improvement on specific targets (e.g. see T2). Notice that more localized calibration approaches do not significantly affect the target gain values. Finally, it is worth noting that analogous considerations can be made also for the real target (Delphin). This clearly proves the effectiveness of the considered processing scheme and the benefits of the proposed approaches for adaptive channel calibration.

Non-negligible clutter residuals are still visible in the final map. In particular, they are associated to backscattered echoes from forested areas close to receiver position and they are probably due to ICM limitations or potential additional error sources. However, these contributions have been 
() 2020 IEEE. Personal use of this material is permitted. Permission from IEEE must be obtained for all other uses, in any current or future media, including reprinting/republishing this material for advertising or promotional purposes, creating new collective works, for resale or redistribution to servers or lists, or reuse of any copyrighted component of this work in other works. DOI: 10.1109/TAES.2020.2987478.

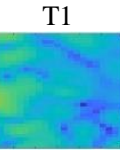

$5.3 \mathrm{~dB}$
$\mathrm{T} 2$

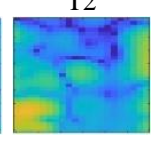

$1.6 \mathrm{~dB}$
T3

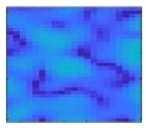

$8.3 \mathrm{~dB}$

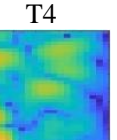

$3.3 \mathrm{~dB}$

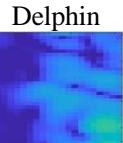

$2.1 \mathrm{~dB}$

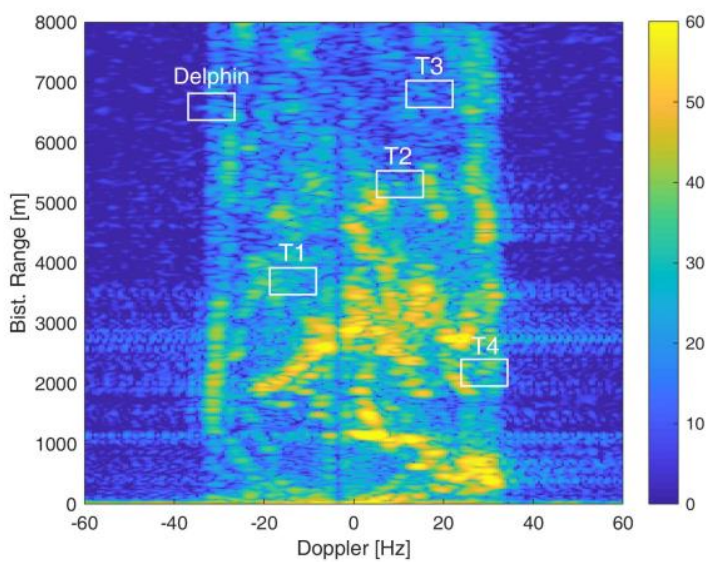

(a)

$\mathrm{T} 1$

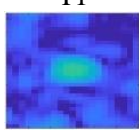

$25.6 \mathrm{~dB}$
$\mathrm{T} 2$

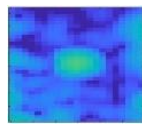

$19.5 \mathrm{~dB}$
T3

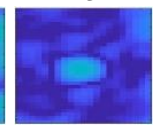

$20.2 \mathrm{~dB}$

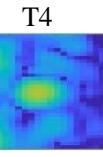

$17.9 \mathrm{~dB}$
Delphin

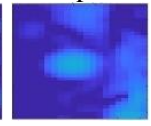

$15.5 \mathrm{~dB}$

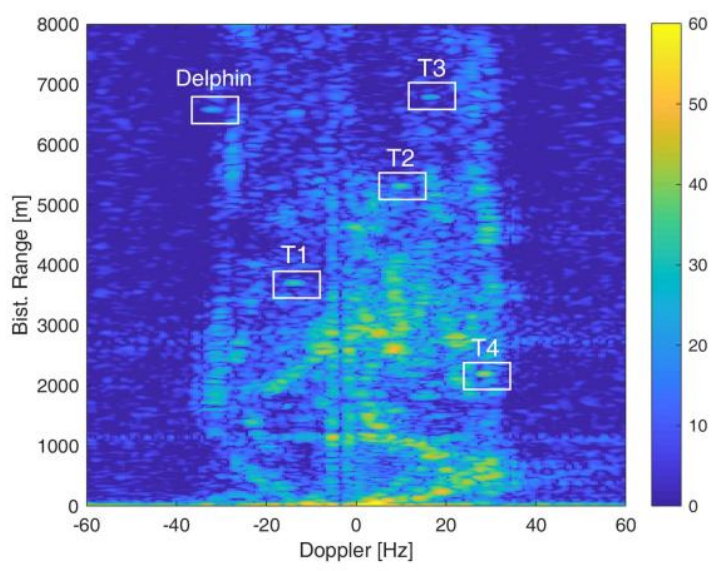

(b)

Fig. 20. Range-Doppler map in presence of real and synthetic targets: (a) before DPCA subtraction; (b) after DPCA subtraction. DDC-RB approach is adopted over range bands of $1600 \mathrm{~m}$. Enlarged views of targets and corresponding SCNR values are reported above.

considerably attenuated compared to their original power level. It is also worth recalling that a very simple architecture and undemanding processing have been used, based on just two receiving channels and DPCA approach, which limits the adaptivity only to the channel calibration stage.

TABLE III. TARGET PARAMETERS

\begin{tabular}{|c|c|c|c|c|c|}
\hline Target & T1 & T2 & T3 & T4 & Delphin \\
\hline$R_{b}$ & $3700 \mathrm{~m}$ & $5300 \mathrm{~m}$ & $6800 \mathrm{~m}$ & $2200 \mathrm{~m}$ & $6596 \mathrm{~m}$ \\
\hline$v_{b}$ & $-6 \mathrm{~m} / \mathrm{s}$ & $9 \mathrm{~m} / \mathrm{s}$ & $6 \mathrm{~m} / \mathrm{s}$ & $10 \mathrm{~m} / \mathrm{s}$ & $-24.5 \mathrm{~m} / \mathrm{s}$ \\
\hline$\varphi_{t}$ & $90^{\circ}$ & $110^{\circ}$ & $85^{\circ}$ & $80^{\circ}$ & $37^{\circ}$ \\
\hline
\end{tabular}

TABLE IV. PERFORMANCE RESUlTS

\begin{tabular}{|c|c|c|c|c|c|}
\hline Target & T1 & T2 & T3 & T4 & Delphin \\
\hline \multicolumn{7}{|c|}{ DSI-based Calibration } \\
\hline$G$ & $-0.3 \mathrm{~dB}$ & $1.2 \mathrm{~dB}$ & $2.8 \mathrm{~dB}$ & $1.5 \mathrm{~dB}$ & $1.8 \mathrm{~dB}$ \\
\hline$C R$ & $5.5 \mathrm{~dB}$ & $9.7 \mathrm{~dB}$ & $4.5 \mathrm{~dB}$ & $4.9 \mathrm{~dB}$ & $2.0 \mathrm{~dB}$ \\
\hline$I F$ & $5.2 \mathrm{~dB}$ & $10.9 \mathrm{~dB}$ & $7.4 \mathrm{~dB}$ & $6.4 \mathrm{~dB}$ & $3.8 \mathrm{~dB}$ \\
\hline \multicolumn{7}{|c|}{ Single Calibration Coefficient } \\
\hline$G$ & $1.5 \mathrm{~dB}$ & $1.9 \mathrm{~dB}$ & $1.9 \mathrm{~dB}$ & $2.5 \mathrm{~dB}$ & $2.9 \mathrm{~dB}$ \\
\hline$C R$ & $16.1 \mathrm{~dB}$ & $12.1 \mathrm{~dB}$ & $8.0 \mathrm{~dB}$ & $10.8 \mathrm{~dB}$ & $6.8 \mathrm{~dB}$ \\
\hline$I F$ & $17.6 \mathrm{~dB}$ & $14.0 \mathrm{~dB}$ & $10.0 \mathrm{~dB}$ & $13.2 \mathrm{~dB}$ & $9.7 \mathrm{~dB}$ \\
\hline \multicolumn{7}{|c|}{ Robust Doppler Dependent Calibration } \\
\hline$G$ & $1.1 \mathrm{~dB}$ & $1.4 \mathrm{~dB}$ & $2.6 \mathrm{~dB}$ & $2.4 \mathrm{~dB}$ & $2.5 \mathrm{~dB}$ \\
\hline$C R$ & $17.9 \mathrm{~dB}$ & $12.6 \mathrm{~dB}$ & $8.2 \mathrm{~dB}$ & $12.4 \mathrm{~dB}$ & $10.0 \mathrm{~dB}$ \\
\hline$I F$ & $19.0 \mathrm{~dB}$ & $14.0 \mathrm{~dB}$ & $10.8 \mathrm{~dB}$ & $14.8 \mathrm{~dB}$ & $12.6 \mathrm{~dB}$ \\
\hline \multicolumn{7}{|c|}{ Robust Doppler Dependent Calibration in Range Bands } \\
\hline$G R$ & $1.1 \mathrm{~dB}$ & $1.5 \mathrm{~dB}$ & $2.5 \mathrm{~dB}$ & $2.5 \mathrm{~dB}$ & $2.5 \mathrm{~dB}$ \\
\hline IF & $19.2 \mathrm{~dB}$ & $16.3 \mathrm{~dB}$ & $9.5 \mathrm{~dB}$ & $12.0 \mathrm{~dB}$ & $10.8 \mathrm{~dB}$ \\
\hline \multicolumn{7}{|c|}{$20.3 \mathrm{~dB}$} & $17.8 \mathrm{~dB}$ & $12.0 \mathrm{~dB}$ & $14.6 \mathrm{~dB}$ & $13.4 \mathrm{~dB}$ \\
\hline
\end{tabular}

\section{CONCLUSIONS}

In this paper we addressed the problem of direct signal interference and clutter cancellation for a passive radar system on moving platform, for the purpose of moving target indication (MTI). A processing scheme was adopted based on reciprocal filtering strategy and DPCA approach. Attention was mainly focused on the development of signal processing algorithms for digital channel calibration, in order to cope with limitation deriving from amplitude and phase inter-channel imbalance.

First, we highlighted the limits of a channel calibration approach based on direct signal, due to the effect of angledependent channel imbalance, for bistatic geometries where DSI and main clutter echoes have different directions of arrival.

Therefore, a two-stage strategy was proposed, aimed at achieving an effective cancellation of both DSI and clutter contributions and removing the influence of DSI on channel calibration. A preliminary suppression of DSI, typically representing the dominant contribution in the range-Doppler map, is provided at both receiving channels by means of an ECA algorithm. Then, a clutter-based channel calibration approach is applied prior to DPCA subtraction, in order to maximize clutter cancellation performance.

Different strategies for digital channel calibration were proposed, based on the criterion of minimizing the output power. Starting from the estimation of a single calibration coefficient (SCC), flexibility of calibration model has been gradually increased, in order to compensate for additional angle (DDC) and range dependent channel errors (DDC-RB). A robust version of these schemes was also introduced to avoid degradation due to the interference of strong targets.

Effectiveness of considered processing scheme and channel calibration approaches have been tested against simulated and experimental data from a DVB-T based mobile PCL system. All the proposed strategies have been shown to provide a significant improvement, with respect to DSI-based 
calibration approach, both in terms of clutter cancellation performance and target detection capability. Specifically, among the proposed techniques, Robust DDC approach proved to yield very good performance against both real and synthetic targets. The Robust DDC-RB approach allowed limited additional improvements in the considered case study and this is mostly related to the adopted acquisition geometry, where a ground-based moving PCL system was employed.

Future research will investigate the applicability of the considered scheme to other mobile PCL system configurations, e.g. airborne receivers. Moreover, efforts will be devoted into the development of space-time techniques (knowledge-based or adaptive) exploiting more than two receiving channels. This on the one hand would allow to improve disturbance cancellation and moving target detection performance, on the other hand it would enable also target angular localization. In fact, multiple spatial degrees of freedom would be required for simultaneous space-time clutter rejection and target DoA estimation. This could be achieved by extending the considered DPCA approach to a multichannel case.

\section{REFERENCES}

[1] P. Lombardo, F. Colone, "Advanced processing methods for passive bistatic radar systems", chapter in book edited by W. L. Melvin and J. A. Scheer, "Principles of Modern Radar: Advanced Radar Techniques," SciTech Publishing, Inc., 2012, pp. 739-821.

[2] J. Palmer, D. Cristallini and H. Kuschel, "Opportunities and current drivers for passive radar research," in IEEE Radar Conference, Johannesburg, 2015.

[3] R. Klemm et alii (Eds.), "Novel radar techniques and applications", Part III: Passive and multistatic radar, IET Publisher, 2017.

[4] J. Brown, K. Woodbridge, H. Griffiths, A. Stove and S. Watts, "Passive bistatic radar experiments from an airborne platform," IEEE Aerospace and Electronic Systems Magazine, pp. 50-55, November 2012

[5] B. Dawidowicz, P. Samczynski, M. Malanowski, J. Misiurewicz and K. S. Kulpa, "Detection of moving targets with multichannel airborne passive radar," IEEE Aerospace and Electronic Systems Magazine, pp. 42-49, 2012

[6] B. Dawidowicz, K. Kulpa, M. Malanowski, J. Misiurewicz, P. Samczynski and M. Smolarczyk, "DPCA detection of moving targets in airborne passive radar," IEEE Transactions on Aerospace and Electronic Systems, pp. 1347-1357, April 2012.

[7] J. Palmer, M. Ummenhofer, A. Summers, G. Bournaka, S. Palumbo and D. Cristallini, "Receiver platform motion compensation in passive radar," IET Radar, Sonar and Navigation, pp. 922-931, 2017.

[8] C. Berthillot, A. Santori, O. Rabaste, D. Poullin, and M. Lesturgie, "BEM reference signal estimation for an airborne passive rada antenna array," IEEE Transactions on Aerospace and Electronic Systems, vol. 53, no. 6, pp. 2833-2845, December 2017

[9] P. Wojaczek, F. Colone, D. Cristallini, P. Lombardo and H. Kuschel, "The application of the Reciprocal Filter and DPCA for GMTI in DVB-T PCL," in International Conference on Radar Systems, Belfast, 2017.

[10] P. Wojaczek, F. Colone, D. Cristallini and P. Lombardo, "ReciprocalFilter-based STAP for passive radar on moving platforms," IEEE Transactions on Aerospace and Electronic Systems, vol. 55, no. 2, pp 967-988, April 2019.

[11] P. Wojaczek and D. Cristallini, "The Influence of Channel Errors in Mobile Passive Radar using DVB-T Illuminators of Opportunity," 19th International Radar Symposium (IRS), Bonn, 2018, pp. 1-10.
[12] P. Wojaczek, D. Cristallini and F. Colone, "Estimating the MV power spectrum for improved clutter suppression in PCL on moving platforms," in IEEE Radar Conference, Boston, 2019.

[13] G. P. Blasone, F. Colone, P. Lombardo, P. Wojaczek and D. Cristallini, "A two-stage approach for direct signal and clutter cancellation in passive radar on moving platforms," in IEEE Radar Conference, Boston, 2019

[14] Klemm, R.: "Principles of Space-Time Adaptive Processing," 3rd edn., IET, 2002.

[15] J. R. Guerci, "Space-Time Adaptive Processing for Radar," Artech House radar library, 2003.

[16] F. Le Chevalier, L. Savy and F. Durniez, "Clutter calibration for space-time airborne MTI radars," Proceedings of International Radar Conference, Beijing, China, 1996, pp. 82-85.

[17] M. A. Koerber and D. R. Fuhrmann, "Radar Antenna Calibration Using Range-Doppler Data," IEEE Seventh SP Workshop on Statistical Signal and Array Processing, Quebec, City, QC, Canada, 1994, pp. 441-444.

[18] F. C. Robey, D. R. Fuhrmann and M. A. Koerber, "Array calibration and modelling of steering vectors," Conference Record of Thirty-Fifth Asilomar Conference on Signals, Systems and Computers (Cat. No.01CH37256), Pacific Grove, CA, USA, 2001, pp. 1121-1126 vol.2.

[19] E. H. Attia and B. D. Steinberg, "Self-cohering large antenna arrays using the spatial correlation properties of radar clutter," in IEEE Transactions on Antennas and Propagation, vol. 37, no. 1, pp. 30-38, Jan. 1989.

[20] C. N. Franz and R. A. Scholtz, "Multipath interferometry technique for airborne array calibration," in IEEE Transactions on Aerospace and Electronic Systems, vol. 35, no. 4, pp. 1369-1382, Oct. 1999.

[21] C. D. Richmond, "The theoretical performance of a class of spacetime adaptive detection and training strategies for airborne radar," Conference Record of Thirty-Second Asilomar Conference on Signals, Systems and Computers (Cat. No.98CH36284), Pacific Grove, CA, 1998, pp. 1327-1331 vol.2.

[22] R. S. Blum and K. F. McDonald, "Analysis of STAP algorithms for cases with mismatched steering and clutter statistics," in IEEE Transactions on Signal Processing, vol. 48, no. 2, pp. 301-310, Feb. 2000.

[23] A. Liu, H. Sun, K. C. Teh, C. J. Baker and C. Gao, "Robust spacetime adaptive processing for nonhomogeneous clutter in the presence of model errors," in IEEE Transactions on Aerospace and Electronic Systems, vol. 52, no. 1, pp. 155-168, February 2016.

[24] J. Palmer, H. Harms, S. Searle and L. Davis, "DVB-T Passive Radar Signal Processing," IEEE Transactions on Signal Processing, pp. 21162126, 15 April 2013.

[25] S. Searle, J. Palmer, L. Davis, D. W. O'Hagan and M. Ummenhofer, "Evaluation of the ambiguity function for passive radar with OFDM transmissions," in IEEE Radar Conference, Cincinnati, 2014.

[26] G. Gassier et al "A Unifying Approach for Disturbance Cancellation and Target Detection in Passive Radar Using OFDM," IEEE Transactions on Signal Processing Vol. 64, Issue 22, pp 5959-5971, 2016.

[27] J. Heckenbach, H. Kuschel, J. Schell and M. Ummenhofer, "Passive radar based control of wind turbine collision warning for air traffic PARASOL," 2015 16th International Radar Symposium (IRS), Dresden, 2015, pp. 36-41. doi: 10.1109/IRS.2015.7226394

[28] W. Shrader and V. G. Hansen, "MTI Radar," in Radar Handbook, M. I. Skolnik, Third Edition, McGraw-Hill, 2008.

[29] M. A. Richards, "Fundamentals of Radar Signal Processing," McGraw-Hill, 2005, pp. 244-250

[30] F. Colone, D. W. O'Hagan, P. Lombardo and C. J. Baker, "A Multistage Processing Algorithm for Disturbance Removal and Target Detection in Passive Bistatic Radar," IEEE Transactions on Aerospace and Electronic Systems, pp. 698-722, April 2009.

[31] F. Colone, C. Palmarini, T. Martelli, and E. Tilli, "Sliding extensive cancellation algorithm for disturbance removal in passive radar," IEEE Transactions on Aerospace and Electronic Systems, vol. 52, no. 3, pp. 1309-1326, June 2016

[32] C. Schwark and D. Cristallini, "Advanced multipath clutter cancellation in OFDM-based passive radar systems," in IEEE Radar Conference (RadarConf), Seattle, 2016. 
[33] J. Ender, "The airborne experimental multi-channel SAR system AERII," in Proc. Eur. Conf. Synth. Aperture Radar (EUSAR), March 1996, pp. 49-52.

[34] C. H. Gierull, "Digital channel balancing of along-track interferometric SAR data," Defence Research and Development Canada (DRDC), Tech. Rep. TM-2003-024, 2003.

[35] S. Baumgartner, M. Gabele, N. Gebert, R. Scheiber, G. Krieger, K. H. Bethke, A. Moreira, "Digital Beamforming and Traffic Monitoring
Using the new FSAR System of DLR," in International Radar Symposium (IRS), Cologne, 2007.

[36] E. Makhoul, S. Baumgartner, M. Jäger, A. Broquetas, "Multichannel SAR-GMTI in Maritime Scenarios With F-SAR and TerraSAR-X Sensors," IEEE Journal of Selected Topics in Applied Earth Observations and Remote Sensing, vol. 8, no. 11, pp. 5052-5067, Nov. 2015.
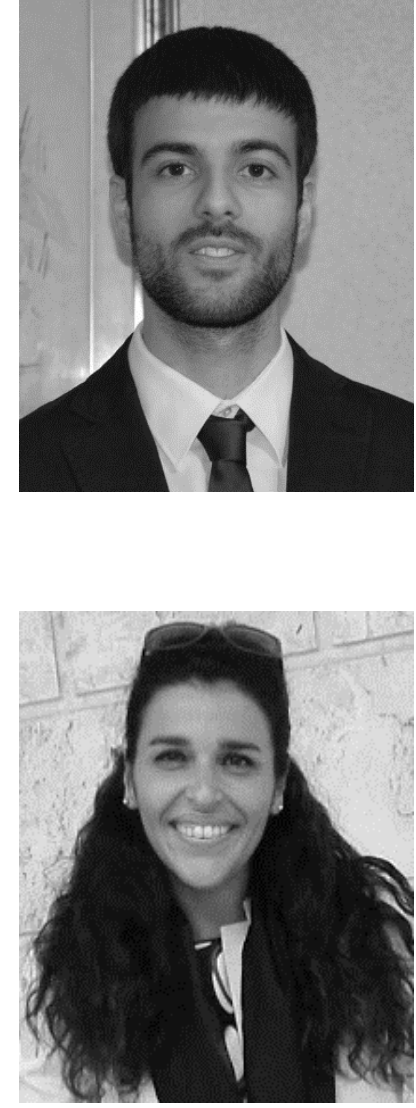

Giovanni Paolo Blasone received the B.Sc. degree (cum laude) in Electronic Engineering and the M.Sc. degree (cum laude) in Communication Engineering, from Sapienza University of Rome, Italy, in 2012 and 2016, respectively. He is currently working towards the Ph.D. degree in Radar and Remote Sensing at the Department of Information Engineering, Electronics and Telecommunications of Sapienza University of Rome.

His main research interests include adaptive signal processing for active and passive multichannel radar systems. He has been involved in research projects funded by the Italian Space Agency and the radar industry.

Fabiola Colone received the laurea degree (B.S.+M.S.) in Telecommunications Engineering and the Ph.D. degree in Remote Sensing from Sapienza University of Rome, Italy, in 2002 and 2006, respectively. She joined the DIET Dept. (formerly INFOCOM) of Sapienza University of Rome as a Research Associate in January 2006. From December 2006 to June 2007, she was a Visiting Scientist at the Electronic and Electrical Engineering Dept. of the University College London, London, U.K. She is currently an Associate Professor at the Faculty of Information Engineering, Informatics, and Statistics of Sapienza University of Rome.

The majority of Dr. Colone's research activity is devoted to radar systems and signal processing. She has been involved, with scientific responsibility roles, in research projects funded by the European Commission, the European Defence Agency, the Italian Space Agency, the Italian Ministry of Research, and the radar industry. Her research has been reported in over 120 publications in international technical journals, book chapters, and conference proceedings. Dr. Colone has been co-recipient of the 2018 Premium Award for Best Paper in IET Radar, Sonar \& Navigation.

Since 2017 she is member of the Board of Governors of the IEEE Aerospace and Electronic System Society (AESS) in which she is currently serving as Vice-President for Member Services, and Editor in Chief for the IEEE AESS QEB Newsletters. She is IEEE Senior Member from 2017 and member of the IEEE AESS Radar System Panel from 2019. Dr. Colone is Associate Editor for the IEEE Transactions on Signal Processing and member of the Editorial Board of the Int. Journal of Electronics and Communications (Elsevier). She served in the technical committee of many international conferences. She was in the organizing committee, as the Student Forum Co-Chair, of the IEEE 2008 Radar Conference (Rome, Italy), and she is currently in the organizing committee, as Special Sessions Co-Chair, of the IEEE 2020 Radar Conference (Florence, Italy). She is also Technical co-Chair of the IEEE Radar Conference 2021 (Atlanta, USA). 

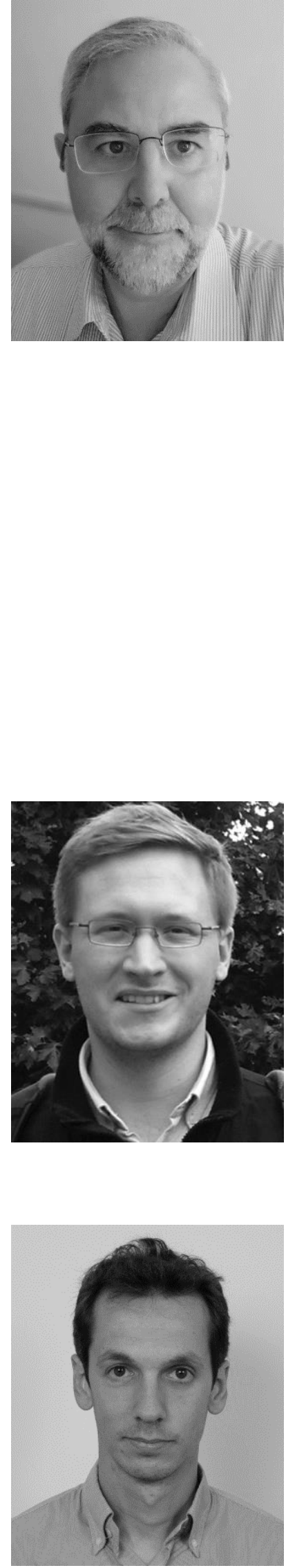

Pierfrancesco Lombardo received the degree in Electrical Engineering and the Ph.D. from the University of Rome "La Sapienza", Italy, respectively in in 1991 and 1995. After serving at the Official Test Center of the Italian Air Force in 1992, he was associate at Birmingham University (UK) and at Defense Research Agency in Malvern in 1994. In 1995 he was research associate at Syracuse University (NY-USA). In 1996 he joined the University of Rome "La Sapienza", where he is presently Full Professor.

Dr. Lombardo is involved in, and coordinates, research projects funded by European and National Research Agencies and national industries. He leads the "Radar, Remote Sensing and Navigation" (RRSN) group at the University of Rome "La Sapienza". He chairs the Cosmo-SkyMed consulting group for the Italian Space Agency. His main interests are radar adaptive signal processing, radar clutter modelling, radar coherent detection, passive radar and multistatic radar, SAR processing and radio-localization systems.

Dr. Lombardo's research has been reported in over 280 publications in international technical journals and conferences and five book chapters. He is co-recipient of the Barry Carlton award (best paper) of IEEE Trans. on AES for year 2001 and of the best paper award for the IEEE Trans. on Geoscience and Remote Sensing for year 2003.

He served in the technical committee of many international conferences on radar systems and signal processing. He was Technical Committee Chairman of the IEEE/ISPRS Workshop on Remote Sensing and Data Fusion over Urban Areas URBAN'2001, Rome, URBAN'2003, Berlin, and URBAN'2005, Tempe (US). He was also Technical Chairman of the IEEE Radar Conference 2008.

Dr. Lombardo is associate Editor for Radar Systems for the IEEE Transactions on Aerospace and Electronic Systems (AES) since June 2001, Technical Editor for Radar System since January 2016 and presently Associate Editor in Chief. He is member of the IEEE AES Radar System Panel and the Editorial board of IET Proceedings on Radar Sonar and Navigation.

Philipp Wojaczek received the M.Sc. degree in electrical engineering, electronics and information technology from the Friedrich-Alexander-Universität Erlangen-Nürnberg, Erlangen, Germany, in 2014, and the Ph.D. degree in radar remote sensing from the University of Rome "La Sapienza," Rome, Italy, in 2019, respectively.

Since 2015, he has been a Research Scientist with the Department for Passive Radar and Anti-Jamming Techniques, Fraunhofer Institute for High Frequency Physics and Radar Techniques FHR, Wachtberg, Germany. His research interests are on signal processing for passive radar systems. He is recipient of the Robert T. Hill Best Dissertation Award for his Ph.D. thesis awarded from the IEEE Aerospace and Electronic Systems Society in 2019.

Diego Cristallini was born in Terni, Italy, in 1981. He graduated cum laude in Telecommunication Engineering in May 2006 from the University of Rome "La Sapienza". In April 2010 he received the Ph.D. degree in Radar Remote Sensing also from the University of Rome "La Sapienza" with a thesis entitled "Innovative adaptive techniques for multi-channel spaceborne SAR systems". From December 2009 to February 2015 he has been with the Array-based Radar Imaging Department of the Fraunhofer Institute for High Frequency Physics and Radar Techniques FHR in Wachtberg, Germany. During this time, he has been working on the development of innovative space-time adaptive techniques for clutter suppression in multi-channel airborne radar systems, with special attention to bistatic systems and to highly non-homogeneous clutter environments. Since March 2015, he is leading the Team on Passive Covert Radar in the Passive Radar and Anti-Jamming Techniques Department of Fraunhofer FHR, Germany.

Dr. Cristallini is co-chair of the NATO-SET 242 group on "PCL on moving platforms" and he has been lecturing for the NATO LS-243 "Passive Radar Technology". He received the Best Paper Award at EUSAR 2014 and co-authored the Best Poster Award at EUSAR 2018. 